This article has been accepted for publication in Monthly Notices of the Royal Astronomical Society (C: 2021 The Authors. Published by Oxford University Press on behalf of the Royal Astronomical Society. All rights reserved. 


\title{
Simulations and observational tests of primordial magnetic fields from Cosmic Microwave Background constraintsok
}

\author{
F. Vazza ${ }^{\circledR}, 1,2,3 \star$ D. Paoletti, ${ }^{4,5}$ S. Banfi, ${ }^{1,3}$ F. Finelli, ${ }^{4,5}$ C. Gheller, ${ }^{3}$ S. P. O’Sullivan ${ }^{\circledR 6}$ and M. Brüggen ${ }^{\circledR 2}$ \\ ${ }^{1}$ Dipartimento di Fisica e Astronomia, Universitá di Bologna, Via Gobetti 92/3, I-40121 Bologna, Italy \\ ${ }^{2}$ Hamburger Sternwarte, Gojenbergsweg 112, D-21029 Hamburg, Germany \\ ${ }^{3}$ INAF, Istituto di Radio Astronomia, Via Gobetti 101, I-40129 Bologna, Italy \\ ${ }^{4}$ INAF, Osservatorio di Astrofisica e Scienza dello Spazio, Via Gobetti 101, I-40129 Bologna, Italy \\ ${ }^{5}$ INFN, Sezione di Bologna, Via Irnerio 46, I-40126 Bologna, Italy \\ ${ }^{6}$ School of Physical Sciences and Centre for Astrophysics \& Relativity, Dublin City University, Glasnevin D09 W6Y4, Ireland
}

Accepted 2020 November 3. Received 2020 November 3; in original form 2020 July 30

\begin{abstract}
We present the first cosmological simulations of primordial magnetic fields derived from the constraints by the Cosmic Microwave Background observations, based on the fields' gravitational effect on cosmological perturbations. We evolved different primordial magnetic field models with the ENZO code and compared their observable signatures (and relative differences) in galaxy clusters, filaments, and voids. The differences in synchrotron radio powers and Faraday rotation measure from galaxy clusters are generally too small to be detected, whereas differences present in filaments will be testable with the higher sensitivity of the Square Kilometre Array. However, several statistical full-sky analyses, such as the cross-correlation between galaxies and diffuse synchrotron power, the Faraday rotation structure functions from background radio galaxies, or the analysis of arrival direction of ultra-high-energy cosmic rays, can already be used to constrain these primordial field models.
\end{abstract}

Key words: methods: numerical-intergalactic medium-large-scale structure of Universe.

\section{INTRODUCTION}

Although we understand by and large how the inhomogeneities of the cosmic microwave background (CMB) at $z \sim 1100$ are related to the distribution of dark (DM) and baryonic matter, the origin of extragalactic magnetic fields is still a puzzle (e.g. Widrow 2002; Ryu et al. 2012; Widrow et al. 2012). The magnetic fields observed in galaxies (e.g. Beck et al. 2012; Pakmor, Marinacci \& Springel 2014; Rieder \& Teyssier 2016, 2017; Seta \& Beck 2019; Sharda, Federrath \& Krumholz 2020, for recent work on the subject), or in the intracluster medium (e.g. Dolag, Bartelmann \& Lesch 1999; Brüggen et al. 2005; Ryu et al. 2008; Xu et al. 2009; Beresnyak \& Miniati 2016a), are likely to be the result of amplification of weak seed fields (e.g. Donnert et al. 2018, for recent reviews). However, it remains unclear whether such seeds were already present at the epoch of the CMB, or whether they arose during galaxy formation, through magnetized winds and jets.

Several mechanisms to generate primordial seed fields have been suggested. These may either involve inflation or take place in the post-inflationary epoch, with the latter referred historically to as causal generation mechanisms. Causal magnetic fields can be possibly generated with large amplitudes, but suffer from small coherence lengths (with the size of the Hubble radius at the generation time being the maximum) and they thus require inverse cascade mechanisms to transfer energy to the largest scales. Among causal generation mechanisms, those associated with phase transitions as

^E-mail: franco.vazza2@unibo.it the electroweak or the QCD (e.g. Quashnock, Loeb \& Spergel 1989; Vachaspati 1991; Baym, Bodeker \& McLerran 1996; Sigl, Olinto \& Jedamzik 1997; Hindmarsh \& Everett 1998; Grasso \& Riotto 1998; Ahonen \& Enqvist 1998; Boyanovsky \& de Vega 2005; Caprini, Durrer \& Servant 2009; Tevzadze et al. 2012; Zhang, Vachaspati \& Ferrer 2019; Ellis et al. 2019) are extremely important, but they require a first-order phase transition, which is currently disfavoured. Alternative causal mechanisms involve second-order perturbations via the Harrison mechanism and recombination, but these can generate only very weak final fields (Fenu, Pitrou \& Maartens 2011; Matarrese et al. 2005; Fidler, Pettinari \& Pitrou 2016). A common characteristics of causal generation mechanisms is that the magnetic fields they produce have a scale dependence, with a spectral index equal or greater than 2 (Durrer \& Caprini 2003).

On the contrary, inflation can generate magnetic fields with different coherence lengths and scale dependencies (however, in this work we assume -2.9 as minimal index to avoid infrared divergences in the energy-momentum tensor of the fields). Magnetic fields can be generated during inflation by breaking the conformal invariance for the electromagnetic field, or by coupling it to other light fields (e.g. Turner \& Widrow 1988; Ratra 1992; Giovannini \& Shaposhnikov 2000; T"ornkvist et al. 2001; Bamba \& Yokoyama 2004; Ashoorioon \& Mann 2005; Demozzi, Mukhanov \& Rubinstein 2009; Kanno, Soda \& Watanabe 2009; Caldwell, Motta \& Kamionkowski 2011; Jain, Durrer \& Hollenstein 2014; Fujita et al. 2015), and depending on the specific mechanism the resulting fields have different characteristics. Such primordial seed fields are found to produce either small ( $\leq$ Mpc, e.g. Chernin 1967), or large (e.g. Zel'dovich 1970; Turner \& Widrow 1988) coherence lengths, which may still persist today (e.g. 
Hutschenreuter et al. 2018), at least in the emptiest cosmic regions. Primordial magnetic fields can also possibly carry information on the generation of primordial helicity (e.g. Semikoz \& Sokoloff 2005; Campanelli 2009; Kahniashvili, Brandenburg \& Tevzadze 2016). The subsequent amplification of these seed fields, plausibly by the dynamo mechanism (see Federrath 2016; Donnert et al. 2018, for recent reviews), further adds to the theoretical predictions of the primordial generation mechanisms.

Several different observations can be used to constrain primordial magnetic fields generated prior to big bang nucleosynthesis. Magnetic fields with a primordial origin, modelled as a stochastic background, can be probed by the anisotropy pattern of the CMB. The analysis of the gravitational effect on the $\mathrm{CMB}$ anistropies angular power spectra in temperature and polarization with recent Planck (Akrami et al. 2018), BICEP/Keck Array (Ade et al. 2018), SPT (Keisler et al. 2015) data strongly constrains fields with amplitude values larger than a few co-moving $\sim$ nG on $\sim$ Mpc scales (Planck Collaboration XIX 2016; Zucca, Li \& Pogosian 2017; Paoletti \& Finelli 2013, 2011, 2019; Shaw \& Lewis 2012). Corresponding bounds have been also derived for an homogeneous primordial magnetic field, whose main additional effect is the breaking of isotropy, that is constrained to very few $\mathrm{nG}$ already with $C O B E$ satellite data (Barrow, Ferreira \& Silk 1997). However, these bounds may be slightly relaxed in the presence of free-streaming neutrinos (Adamek et al. 2011). On the other hand, magnetic fields in cosmic voids are constrained to be larger than the lower limits deduced by the absence of an Inverse Compton Cascade from distant blazars (e.g. Dolag et al. 2009; Neronov \& Vovk 2010; Dolag et al. 2011; Arlen et al. 2014; Caprini \& Gabici 2015; Chen, Buckley \& Ferrer 2015), of order $\sim 10^{-7} \mathrm{nG}^{1}$ Even more stringent limits on the amplitude of magnetic seed fields have been derived by including post-recombination heating ( Chluba et al. 2015; Kunze \& Komatsu 2015; Paoletti et al. 2019) or by modelling the small-scale baryonic density fluctuation induced by primordial magnetic fields, leading to inhomogeneous recombination and heating, which would alter the peaks and heights of the large-scale anisotropies of the CMB (Trivedi et al. 2018; Jedamzik \& Saveliev 2019).

Any significant detection of magnetic fields beyond galaxies and galaxy clusters (or even any robust upper limit) would thus help to explore the origin of cosmic magnetism, because several theoretical works have shown that the radio signatures of drastically different magnetic field scenarios would leave very different imprints in Faraday rotation and/or synchrotron emission from the magnetized cosmic web (Donnert et al. 2009; Vazza et al. 2015; Vazza et al. 2017a). Due to the very weak radio signal expected outside of the overdensities typical of haloes and cluster outskirts, different observational proxies have also been proposed, i.e. by studying the propagation of ultra-high-energy cosmic rays (e.g. Dolag et al. 2005; Hackstein et al. 2017), by using the non-detection of Inverse Compton emission from distant blazars. From the latter, lower limits of $\geq 10^{-16} \mathrm{G}$ on $\sim$ Mpc scales have been derived (Dolag et al. 2009; Neronov \& Vovk 2010; Dolag et al. 2011; Arlen et al. 2014; Caprini \& Gabici 2015; Chen et al. 2015), and more stringent limits will be obtained in the near future by the Cherenkov Telescope Array (CTA; Sol et al. 2013; Meyer, Conrad \& Dickinson 2016).

Finally, the presence of significant large-scale magnetic fields has been suggested as a possible explanation for the puzzling lack of infrared absorption in the observed spectra of distant blazars (e.g.

\footnotetext{
${ }^{1}$ See, however, Broderick, Chang \& Pfrommer (2012) for a possible different
} interpretation.
Horns et al. 2012; Tavecchio et al. 2012). Axion-like particles (ALPs) are promising candidate for DM (Raffelt \& Stodolsky 1988; Csáki et al. 2003) and they can oscillate into high-energy photons (and back) in the presence of background magnetic fields, reducing the effective opacity of emitted $\gamma$-ray photons (Horns et al. 2012). With recent work, we simulated the propagation of photons from redshift $z=1$ and computed the expected conversion into ALPs, and we found that photons-ALPs oscillations are possible for lines of sight crossing structures with $\sim 1-10 \mathrm{nG}$ on scales of a few $\sim \mathrm{Mpc}$. (Montanino et al. 2017), again likely to be tested by the upcoming CTA (Montanino et al. 2017).

In this work, we present for the first time cosmological simulations of primordial magnetic fields derived from the constraints from the CMB. By evolving different primordial magnetic field models from $z=40$ to $z=0$, we generated observable signatures across the cosmic web and used them to investigate whether further constraints can be put on primordial scenarios. This paper is structured as follows: after describing our simulations and numerical tools in Section 2, we present our results in Section 3. We provide physical and numerical caveats in Section 4 before we summarize and conclude our work in Section 5.

\section{METHODS}

\subsection{Cosmological simulations}

We used the cosmological Eulerian code ENZO (Bryan et al. 2014) to resimulate a cosmic volume of $(100 \mathrm{Mpc})^{3}$ comoving, with the constant spatial resolution of $\Delta x=195$ comoving kpc, using $512^{3}$ cells and dark matter particles. We investigate the evolution and topology of magnetic fields in the most rarefied cosmic regions, removed from the contamination from galaxy-related processes. In order to make up for the lack of resolution, we apply a subgrid scheme for dynamo amplification (Section 2.1.2).

The adopted cosmology is a Lambda cold dark matter $(\Lambda \mathrm{CDM})$ model with $\Omega_{\mathrm{b}}=0.0468, \Omega_{\mathrm{m}}=0.308, \Omega_{\Lambda}=0.692, H_{0}=$ $67.8 \mathrm{~km}^{-1} \mathrm{~s}^{-1} \mathrm{Mpc}, \sigma_{8}=0.815$, and a spectral index for the initial matter power spectrum of $n=1.0^{2}$ and an initial redshift $z_{\text {ini }}=40$.

\subsubsection{MHD method}

The adopted scheme for magnetohydrodynamics (MHD) is the conservative Dedner formulation (Dedner et al. 2002), which uses hyperbolic divergence cleaning to keep the $\nabla \cdot \vec{B}$ as small as possible, in combination with the piecewise linear method reconstruction (PLM) technique and with the Harten-Lax-Van Leer (HLL) approximate Riemann solver. The time integration is based on the total variation diminishing (TVD) second-order Runge-Kutta (RK) scheme (Shu \& Osher 1988).

The choice of using a constant spatial resolution, rather than an adaptive one, is related to the fact that all effects we are looking for in this project are related to underdense cosmic regions (e.g. voids) or mildly dense structures (e.g. matter sheets, filaments, cluster outskirts), and therefore the use of a fixed spatial resolution is helpful for our analysis. For applications of adaptive mesh refinement for resolving turbulence in the intergalactic medium, we refer the reader to Iapichino, Federrath \& Klessen (2017). Tests on the dependence

\footnotetext{
${ }^{2}$ Due to the little correlation of the scalar spectral index with the primordial magnetic field configuration we expect little dependence on the value of ns of the field final properties.
} 


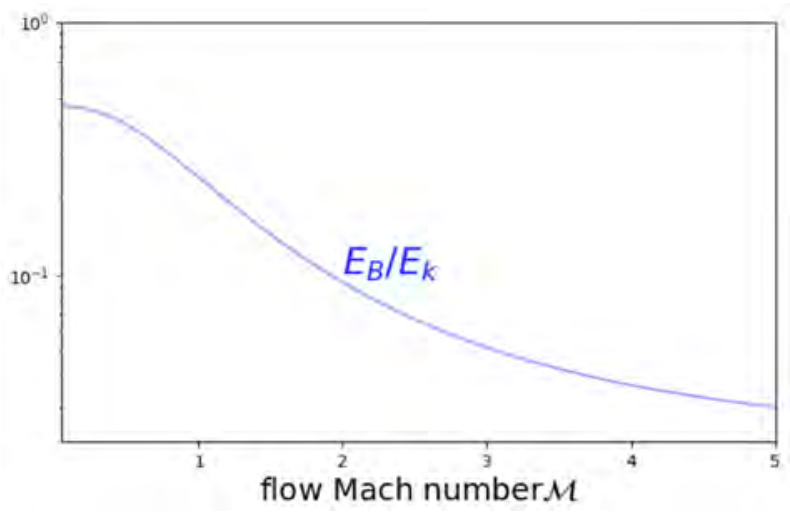

Figure 1. Predicted saturation level of magnetic energy with respect to the turbulent kinetic energy, in the case of solenoidal forcing of turbulence, as computed in Federrath et al. (2014) and adopted in our sub-grid modelling of small-scale dynamo amplification (Section 2.1.2).

of some of our results on the spatial resolution are presented in the Appendix.

\subsubsection{Subgrid dynamo model}

Our simulations are non-radiative, i.e. only the effects of cosmological expansion, gravity, and magnetohydrodynamics are included. To make up for the lack of small-scale dynamo amplification in the turbulent interiors of haloes, we applied a subgrid model for magnetic field amplification (Porter, Jones \& Ryu 2015; Beresnyak \& Miniati 2016a; Wittor, Vazza \& Brüggen 2017a) within overdense regions $(\rho \geq 50\langle\rho\rangle)$ (e.g. Vazza et al. 2017a). At run time we measure the gas vorticity and use it to estimate the dissipation rate of solenoidal turbulence into magnetic field amplification. Our procedure is based on Federrath et al. (2014), and on their fitting formulas to predict the growth of magnetic energy from scales that our simulation cannot directly resolve.

The local velocity enstrophy, $(\nabla \times \vec{v})^{2} \equiv \epsilon_{\omega}$ is a convenient quantity to estimate the turbulent dissipation rate, because the turbulent kinetic energy flux is conserved along the cascade. Following previous work (Jones et al. 2011; Porter et al. 2015; Vazza et al. 2017b; Wittor et al. 2017b), we assume that a small fraction, $\eta_{t} \approx$ $10^{-2}$, of such kinetic power gets channeled into the amplification of magnetic fields, $F_{\text {turb }} \simeq \eta_{t} \rho \epsilon_{\omega}^{3} / L$, where $L$ is the stencil of cells to compute the vorticity. The fraction of turbulent kinetic power that gets converted into magnetic energy, $\epsilon_{\text {dyn }}$, sets the amplified magnetic energy as $E_{\mathrm{B}, \mathrm{dyn}}=\epsilon_{\mathrm{dyn}}(\mathcal{M}) F_{\mathrm{turb}} \Delta t$. For a reasonable guess on $\epsilon_{\mathrm{dyn}}$, we rely on Federrath et al. (2014), who simulated smallscale dynamo in a variety of conditions for the forcing of turbulence. We can thus estimate the saturation level and the typical growth time of magnetic fields as a function of the local Mach number of the flow $(\mathcal{M})$, and set $\epsilon_{\text {dyn }}(\mathcal{M}) \approx\left(E_{B} / E_{k}\right) \Gamma \Delta t$, where $E_{B} / E_{k}$ is the ratio between magnetic and kinetic energy at saturation, and $\Gamma$ is the growth rate, taken from Federrath et al. (2014). In Fig. 1, we show the $E_{B} / E_{k}(\mathcal{M})$ relation adopted in our model, which is derived for a purely solenoidal forcing of turbulence (Federrath et al. 2014), which is appropriate for haloes, given the predominance of solenoidal motions (e.g. Miniati 2014; Vazza et al. 2017b). As a reference value, for a $\mathcal{M}=0.5$ turbulent Mach number, the model predicts saturated level of magnetic energy which is $\approx 40$ per cent of the local solenoidal turbulent kinetic energy. In this approach, we need to specify the topology of the amplified magnetic field. For simplicity, the additional field is taken to be parallel to the local gas vorticity, such that the newly generated field is solenodial by construction. Energy and momentum are conserved assuming an isotropic dissipation of the small-scale velocity vectors. Admittedly, this procedure is simpler than more sophisticated subgrid models (Grete et al. 2016). However, this simplistic method reproduced the results obtained by other methods (e.g. Ryu et al. 2008; Vazza et al. 2017a; Hackstein et al. 2019). While the application of the sub-grid dynamo model adds realism to the magnetic field distribution in our volume, it has no impact on the intermediate and low-density regime in which we study the differences introduced by our CMB-based magnetic fields. As it solely relies on the gas velocity field, which is basically identical in all runs, the contribution from the sub-grid dynamo model within haloes is exactly the same in all models.

As an important caveat to our sub-grid dynamo model, we stress that in reality the dynamo growth rates must depend on the magnetic Prandtl number $\left(P_{M}\right)$, on the kinematic Reynolds number $\left(R_{e}\right)$, as well as on the turbulence driving (Federrath et al. 2011, 2014). For example, Schober et al. (2012) measured the increase of the growth rate with the (numerical) Reynolds number. Furthermore, the Reynolds and the Prandtl numbers in the ICM are expected to be much larger than anything that can be resolved by MHD simulations in the near future (e.g. Brunetti \& Lazarian 2011; Donnert et al. 2018). As usual in cosmological MHD simulations, $P_{M} \approx 1$, i.e. the kinematic and the magnetic Reynolds number are equal as we only have numerical viscosity and resistivity (e.g. Donnert et al. 2018). Of course, given the complexity of plasma physics, this convenient assumption is highly questionable (e.g. Schekochihin et al. 2004; Brunetti \& Lazarian 2011; Beresnyak \& Miniati 2016b). Moreover, our model misses turbulence in the early Universe that develops during the collapse of filaments and which is generated on scales too small to be resolved. In this case, the main driving mode of turbulence should be compressive driving (e.g. Federrath 2018). In this respect, more flexible sub-grid model of dynamo amplification may be explored in the future (e.g. Grete et al. 2017, 2019), even if the main conclusions of our paper, regarding observable properties of cosmic structures on $\geq \mathrm{Mpc}$ scale, are expected to remain unaltered.

\subsubsection{Primordial magnetic field models}

We modelled the primordial magnetic fields using a stochastic background, described by the two-point correlation function (Mack, Kahniashvili \& Kosowsky 2002; Finelli, Paci \& Paoletti 2008)

$$
\left\langle B_{i}^{\star}(\mathbf{k}) B_{j}\left(\mathbf{k}^{\prime}\right)\right\rangle=\delta^{(3)}\left(\mathbf{k}-\mathbf{k}^{\prime}\right) P_{i j}(\hat{\mathbf{k}}) P_{B}(k)(2 \pi)^{3},
$$

where $i$ and $j$ are spatial indices. $\delta^{(3)}\left(\mathbf{k}-\mathbf{k}^{\prime}\right)$ is the Dirac delta function, with unit vector $\hat{k}_{i}=k_{i} / k, P_{i j}(\hat{\mathbf{k}})=\delta_{i j}-\hat{k}_{i} \hat{k}_{j}$ is the operator for transverse plane projection, and $P_{B}(k)$ is the power spectrum of the magnetic field. The fields scale dependence is described with a power-law spectrum: $P_{B}(k)=P_{B 0} k^{\alpha}$ characterized by the amplitude of the fields and the spectral index. As a convention, we describe the amplitude by smoothing the fields on the scale $\lambda$ and using $B_{\lambda}$

$P_{B}(k)=P_{B} k^{\alpha}=\frac{2 \pi^{2} \lambda^{3} B_{\lambda}^{2}}{\Gamma\left(n_{B} / 2+3 / 2\right)}(\lambda k)^{\alpha}$,

where $\lambda$ is a comoving smoothing length (with spatial frequency $k_{\lambda}=$ $2 \pi / \lambda)$, with a Gaussian kernel $\propto \exp \left[-x^{2} / \lambda^{2}\right]$. For $k>k_{D}$, where $k_{D}$ is the cut-off wavenumber, the power spectrum gets dissipated through Alfvén wave damping. The values of $k_{D}$ for each magnetic field model are given in Table 1 (8th column). 
Table 1. List of cosmological ENZO simulations produced for this work.

\begin{tabular}{lccccccccccc} 
Run ID & $\begin{array}{c}\text { Volume } \\
(\mathrm{Mpc})\end{array}$ & $\begin{array}{c}\text { Resolution } \\
(\mathrm{kpc})\end{array}$ & $\Omega_{\mathrm{M}}$ & $\Omega_{\mathrm{b}}$ & $\Omega_{\Lambda}$ & $\sigma_{8}$ & $\begin{array}{c}h \\
\left(100 \mathrm{~km}^{-1} \mathrm{~s}^{-1} \mathrm{Mpc}\right)\end{array}$ & $\begin{array}{c}k_{\mathrm{D}} \\
(1 / \mathrm{Mpc})\end{array}$ & $\begin{array}{c}B_{\mathrm{Mpc}} \\
(\mathrm{nG})\end{array}$ & $\begin{array}{c}B_{\text {eff }} \\
(\mathrm{nG})\end{array}$ & $\begin{array}{c}\text { Description } \\
\text { B0 }\end{array}$ \\
\hline 100 & 195 & 0.308 & 0.0478 & 0.692 & 0.815 & 0.678 & - & 2.0 & 2.0 & Homogeneous \\
B1 & 100 & 195 & 0.308 & 0.0478 & 0.692 & 0.815 & 0.678 & 84.7 & 2.0 & 2.53 & $\alpha=-2.9$ \\
B2 & 100 & 195 & 0.308 & 0.0478 & 0.692 & 0.815 & 0.678 & 25.8 & 1.87 & 47.63 & $\alpha=-1.0$ \\
B3 & 100 & 195 & 0.308 & 0.0478 & 0.692 & 0.815 & 0.678 & 37.6 & 0.35 & 70.1 & $\alpha=0.0$ \\
B4 & 100 & 195 & 0.308 & 0.0478 & 0.692 & 0.815 & 0.678 & 56.6 & 0.042 & 95.21 & $\alpha=1.0$ \\
B5 & 100 & 195 & 0.308 & 0.0478 & 0.692 & 0.815 & 0.678 & 87.9 & 0.003 & 119.21 & $\alpha=2.0$ \\
\hline
\end{tabular}

It is customary to set $\lambda=1 \mathrm{Mpc}$, and therefore in the remainder of the paper we will use $B_{\mathrm{Mpc}}=B_{\lambda}$ to refer to the smoothed magnetic field amplitude.

In Table 1, we provide both the magnetic field settings and the cosmological parameters used in the simulations. The primordial magnetic fields we assume are a bunch of different cases from the range of possible spectral indices, from the almost scale invariant $\alpha=-2.9$ whose energy momentum tensor in Fourier space is infrared dominated, to an high values of $\alpha=2$ which corresponds to the minimum index allowed for causally generated magnetic fields. The values for the amplitudes of the fields are derived by the constraints with the combination of the most recent data of Planck 2018 (Akrami et al. 2018) with ground-based observatory as BICEP/KECK (Ade et al. 2018) and the South Pole Telescope (Keisler et al. 2015) following Paoletti \& Finelli (2019) using the gravitational scalar, vector, and tensor effects of PMFs on $\mathrm{CMB}$ anisotropies. ${ }^{3}$ We stress that, in this work, the magnetic seeds used are modelled with configurations, amplitudes, and corresponding scale dependences, that are directly derived from CMB data constraints. Concerning the homogeneous field case, we assume a value of $2 \mathrm{nG}$ in agreement with the $C O B E$ constraints (Barrow et al. 1997), that still remain the tightest constraints on homogeneous field from $\mathrm{CMB}$ anisotropies. This value is pretty generous and stronger constraints should be provided by Planck in the future, but are not currently available therefore we refer to the $C O B E$ ones. Note that the cosmological parameters are not varied for the different settings of the magnetic fields because, as demonstrated by the constraints derived with different observational data and the forecasts for future experiments (Paoletti \& Finelli 2011, 2013; Planck Collaboration XIX 2016; Paoletti \& Finelli 2019), the inclusion of primordial magnetic fields does not introduce strong degeneracies with the standard cosmological parameters.

We can define an 'effective' magnetic field, corresponding to the magnetic energy density generated by the fields above, $B_{\text {eff }}=\sqrt{\left\langle B^{2}\right\rangle}$ where the latter is the integrated square magnetic field up to $k_{D}$ as in Finelli et al. (2008) and Kahniashvili et al. (2013), it can be computed as

$B_{\mathrm{eff}}=B_{\mathrm{Mpc}} \cdot \frac{\left(k_{D} \lambda\right)^{(\alpha+3) / 2}}{\sqrt{\Gamma(\alpha / 2+5 / 2)}}$.

The $B_{\text {eff }}$ corresponding to each model is given in the 10th column of Table 1: while this is the effective magnetic field value expected if all scales down to $l_{D} \approx 1 / k_{D}$ can be probed, our simulations can only resolve a larger spatial resolution coarser than $l_{D}$.
As a consequence, the effective magnetic field amplitude that can be captured by our simulations will be typically smaller that $B_{\text {eff }}$. In this work, we only consider non-helical magnetic fields. Helical magnetic fields can be generated during inflation Field \& Carroll (2000), Vachaspati (2001), Sigl (2002), Sharma, Subramanian \& Seshadri (2018), and helicity represent an important component in the evolution of the fields as shown by simulations (Christensson, Hindmarsh \& Brandenburg 2001, 2005; Saveliev, Jedamzik \& Sigl 2013; Kahniashvili et al. 2017; Brandenburg \& Kahniashvili 2017). The helicity impact on CMB anisotropies has been investigated in Pogosian, Vachaspati \& Winitzki (2003), Caprini, Durrer \& Kahniashvili (2004), Kunze (2012), Ballardini, Finelli \& Paoletti (2015), Kahniashvili et al. (2014), and the constraints on the helical field amplitude have been explored in Planck Collaboration XIX (2016). Due to the complexity degree required by the presence of an helical component in the magnetic fields, we leave the helicity treatment for a future work.

Here, we impose the initial conditions for the magnetic fields described above at the start of runs, $z_{\text {ini }}=40$. The elapsed time between recombination and $z=40$ the two epochs is $\approx 65 \mathrm{Myr}$, which is negligible compared to the total time of the simulation, and also no significant structure formation is expected to take place in between these two epochs, hence this simplification does not pose any problem for our analysis. ${ }^{4}$

We note that in our analysis we consider only the PMFs as a stochastic background with a given a spectral index and an amplitude compatible with the $\mathrm{CMB}$ anisotropies constraints, leaving the additional impact of magnetized cosmological perturbations on the matter power spectrum (see e.g. Sethi \& Subramanian 2005; Finelli et al. 2008; Shaw \& Lewis 2010; Fedeli \& Moscardini 2012; Kahniashvili et al. 2013) for a future work.

Fig. 2 gives the input magnetic power spectra for our models, while Fig. 3 gives the example of the projected maps of magnetic fields strength at $z=40$ for the B1 $(\alpha=-2.9)$ and for the B4 $(\alpha=1.0)$ models. We remark that the variation of the amplitude with the configuration is central for the results we will comment in the next Sections: spectra that increase the power on the smallest scales are already strongly constrained by the low amplitude of the CMB, whereas fields with more infrared spectra are allowed to larger amplitudes but lower power on much larger scales. The interplay between the scale dependence of the fields, and the constraints on their amplitude by the $\mathrm{CMB}$, will be reflected into the lower redshift constraints explored in the rest of this work.

\footnotetext{
${ }^{3}$ The additional constraints that are not derived in Paoletti \& Finelli (2019) have been computed with the same approach and code as Paoletti \& Finelli (2019).
}

${ }^{4}$ For the current work we do not consider the additional post-recombination effect of PMFs. 


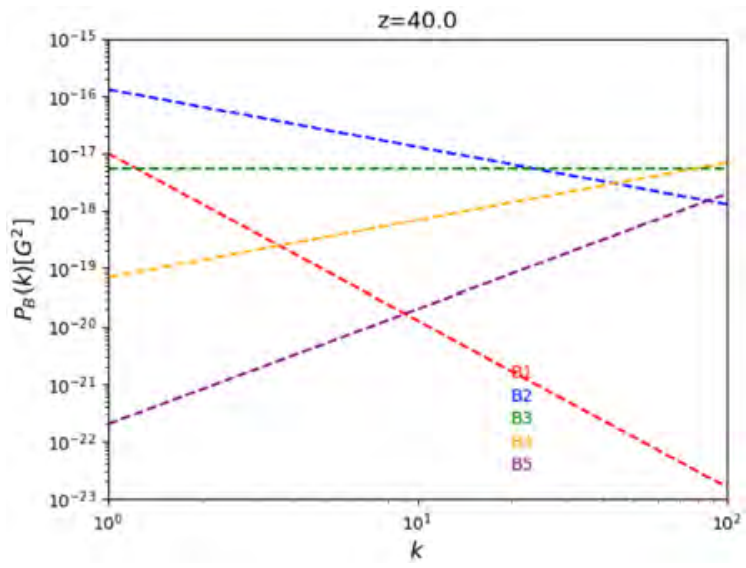

Figure 2. Input 3D power spectra $(z=40)$ of magnetic fields for our models.
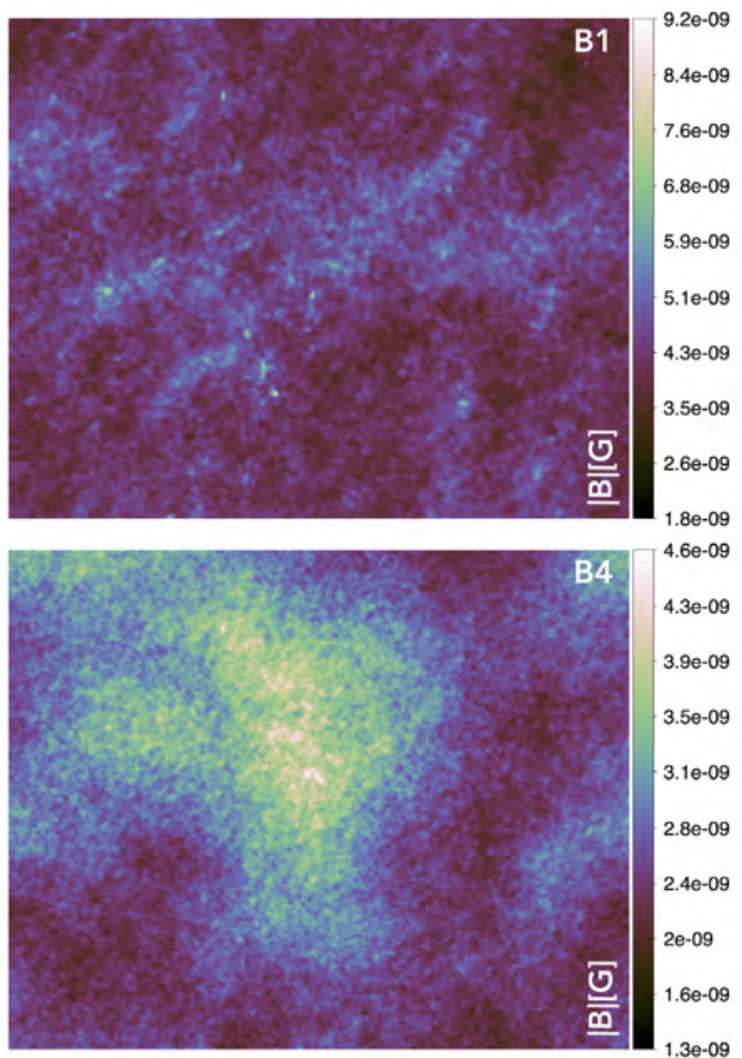

Figure 3. Projected (gas mass-weighted) physical magnetic field strength along the entire $100 \mathrm{Mpc}$ line of sight for run B1 and B4 at the begin of our simulations $(z=40)$.

\section{RESULTS}

\subsection{Properties of simulated magnetic fields}

\subsubsection{Global properties}

The panels in Fig. 4 show the spatial distribution of our simulated magnetic fields, for all models, at $z=1$ and $z=0.02$.

As expected, large differences are present at all epochs, outside of collapsed matter haloes in the volume. The amplitude of magnetic fields in the mildly dense (e.g. sheets/filaments) and underdense (e.g. voids) cosmic environment are found to vary by $\sim 10-10^{2}$ depending on the seeding model, event by the end of the simulation $z=0.02$. The magnetic field amplitude within haloes is instead almost exactly the same in all cases, owing to our subgrid dynamo model, whose key ingredient is the small-scale gas kinetic energy, which is basically identical in all runs.

In Fig. 5, we quantify the typical magnetic field level (and temperature) as a function of gas density. We can notice significant differences among models at low densities, $\rho \leq 10^{-29} \mathrm{~g} \mathrm{~cm}^{-3}$, i.e in the environment of the outer regions of haloes, or even outside them. In voids, there is a $\geq 10$ difference in magnetic field intensity between models. While in the simple uniform seeding model (B0), the magnetization is a strong function of gas density, in most of the other models the presence of initial large fluctuations in the seed fields produces a broader distribution of magnetic fields in voids, smoothing out any strong dependence with density.

A new finding of our analysis is the presence of differences in the average gas temperature in voids $\left(\rho \sim 10^{-32}-10^{-31} \mathrm{~g} \mathrm{~cm}^{-3}\right)$, depending on the magnetic seed model. For example, in models B2, $\mathrm{B} 3$, and $\mathrm{B} 4$ the average temperature of voids can reach $\sim 10^{2}-10^{3} \mathrm{~K}$, i.e. much beyond the $\leq 10 \mathrm{~K}$ of the standard $\mathrm{B} 0$ run here. Both temperatures are unrealistic, in the sense that the reheating from reionization (which would rise the gas temperature to $\sim 10^{3}-10^{4} \mathrm{~K}$ everywhere, Haardt \& Madau 1996) is completely missing in these models. However, such differences are significant and hint at a substantial extra heating that primordial magnetic field fluctuations are introducing. Large initial fluctuations of magnetic field can become relevant for the local gas dynamics, because via induction equation they can trigger the formation of shock waves and the dissipation of turbulent motions into heat, in way qualitatively similar to what has been proposed during recombination epochs (e.g. Trivedi et al. 2018; Jedamzik \& Saveliev 2019). Also post-recombination, dissipative effects on the magnetic fields as MHD decaying turbulence and ambipolar diffusion, whose study is currently based mainly on approximated linear numerical treatments, show similar levels of the heating of the plasma (Chluba et al. 2015; Kunze \& Komatsu 2015; Paoletti et al. 2019). The development of extra shocks for gas with $\rho \leq 10^{-31} \mathrm{~g} \mathrm{~cm}^{-3}$ is well captured by the $(\rho, \mathrm{T})$ phase diagrams of Fig. 6, in which we also computed the dissipated energy flux through shocks identified in the simulation. ${ }^{5}$ The additional shocks in empty regions stand out in the statistics of detected shocks in the left part of the phase diagrams, well away from the typical regime of structure formation shocks driven by gravity (e.g. Vazza et al. 2011).

We remark that such new classes of shocks driven by magnetic fields may not be physically very relevant, because of the aforementioned lack of a reionization heating floor in our model, as well as because the energy flux associated with such shock is extremely small, i.e. $\leq 10^{-7}$ of the total energy dissipation of kinetic energy in the total cosmic volume.

However, the exact topology of magnetic fields in the volume swept by cosmic shocks is also relevant as it can affect the acceleration efficiency of cosmic rays (CR). Protons and/or electrons should undergo different kinds of shock acceleration as a function of plasma parameters as well as of the topology of up-stream magnetic field (e.g. Bykov et al. 2019, and references therein for a recent review). While CR protons should be efficiently accelerated by strong shocks with a quasi-parallel geometry between the shock normal and the upstream magnetic field via diffusive shock acceleration (DSA), CR

\footnotetext{
${ }^{5}$ Shocks are identified in post-processing, with a velocity-based scheme to compute the Mach number based on jumps of thermodynamical quantities, as in Vazza, Brunetti \& Gheller (2009) and Banfi, Vazza \& Wittor (2020).
} 

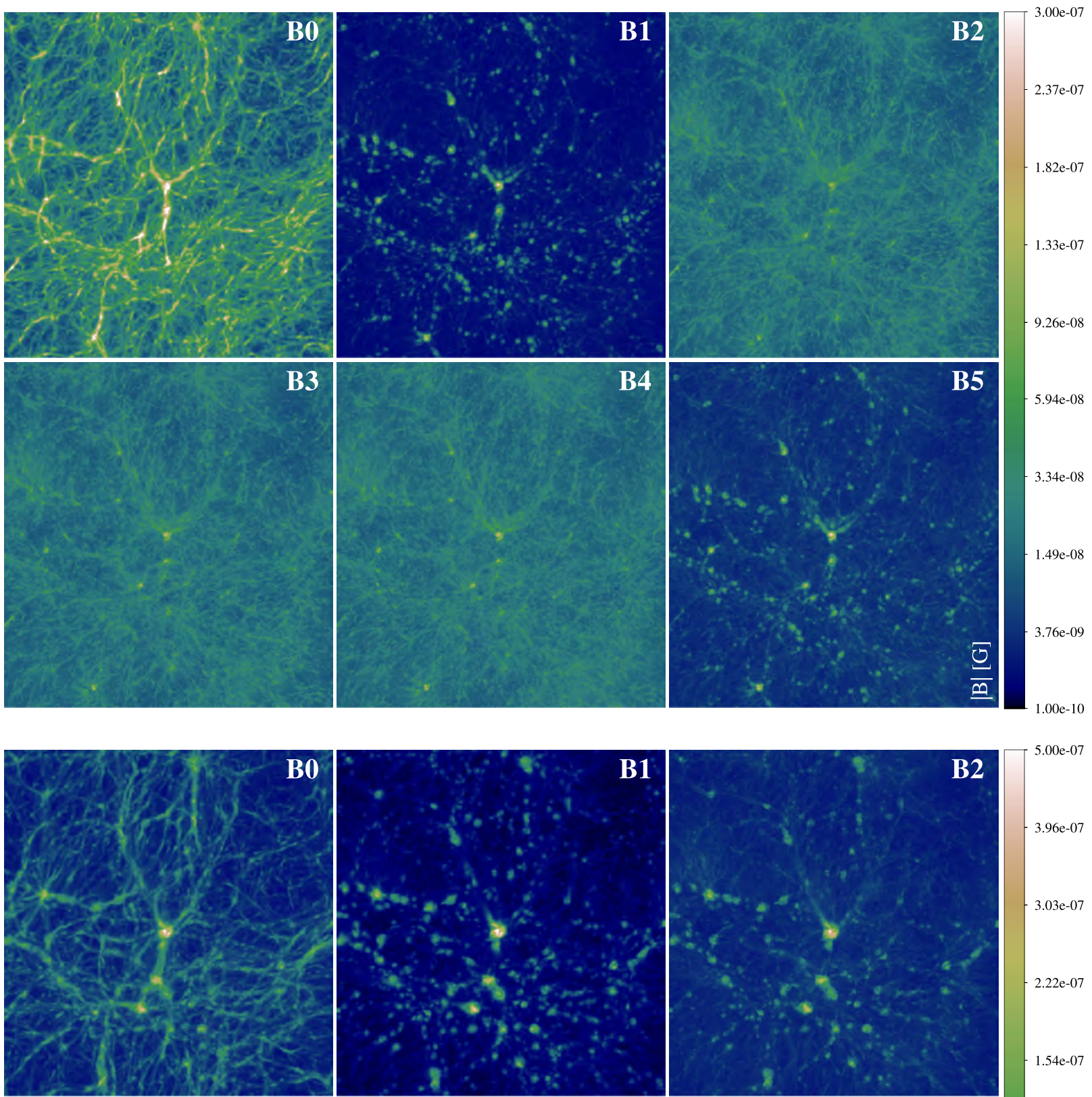

$1.00 \mathrm{e}-10$
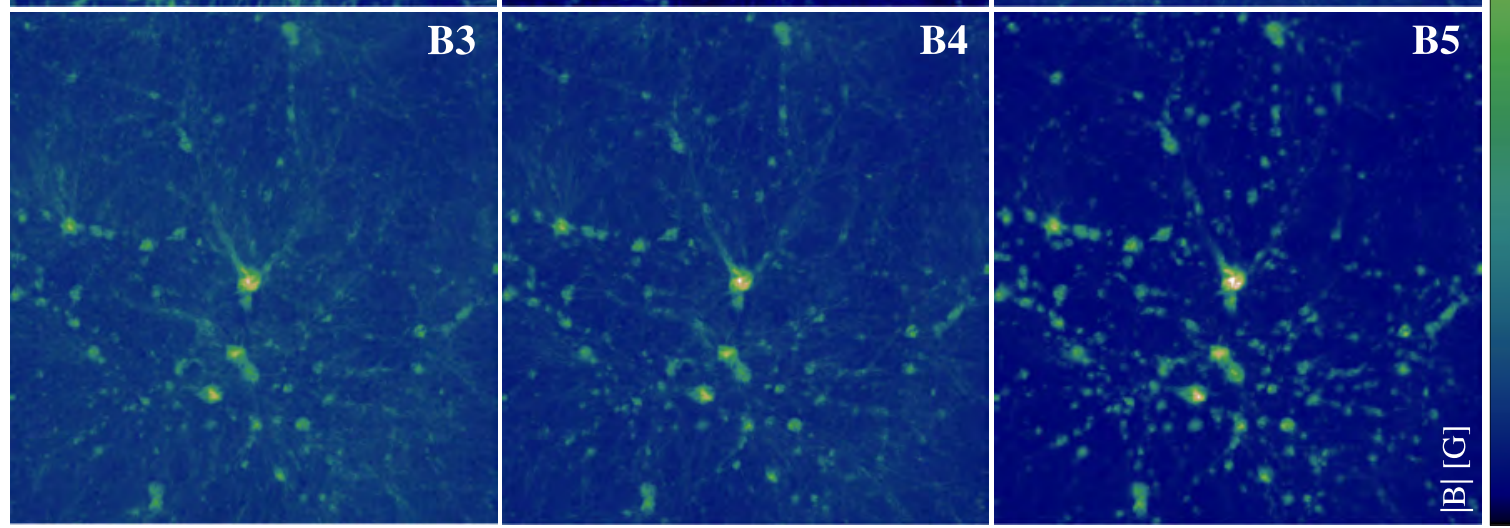

$9.90 \mathrm{e}-08$

$2.22 \mathrm{e}-07$

$1.54 \mathrm{e}-07$

$5.56 \mathrm{e}-08$

$2.47 e-08$

6.20e-09

Figure 4. Projected (gas mass-weighted) proper magnetic field strength along the entire $100 \mathrm{Mpc}$ line of sight, for all our models at $z=1$ (top) and at $z=0$ (bottom). 

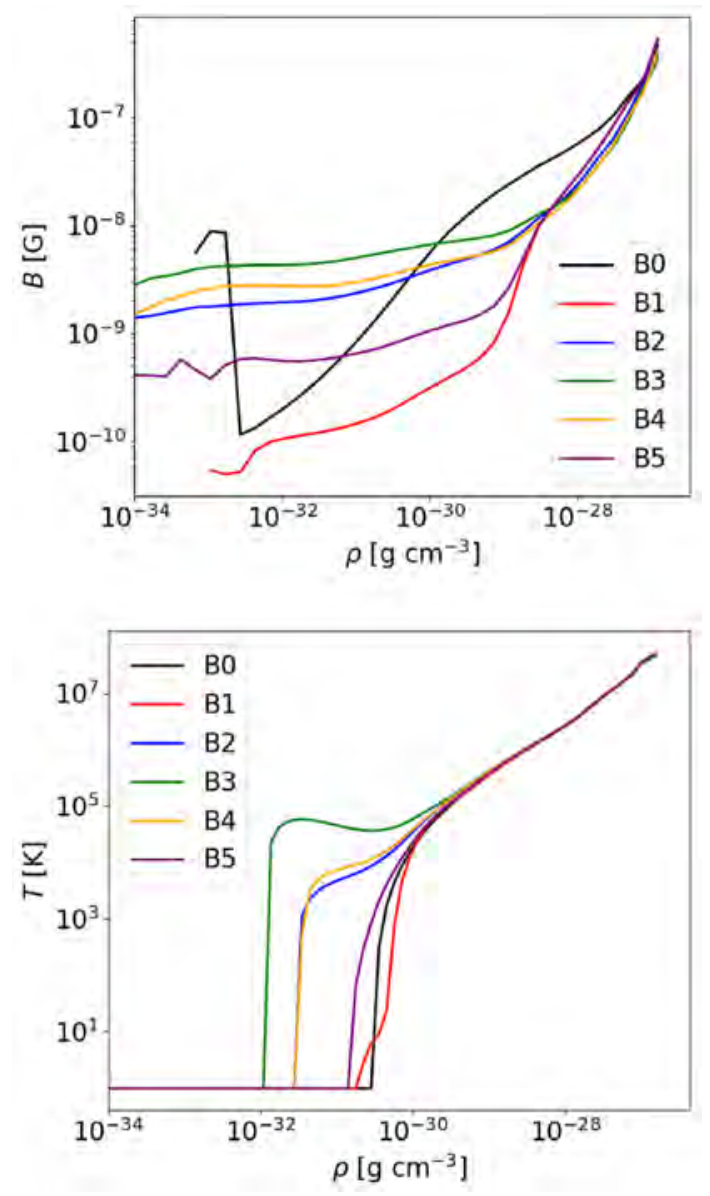

Figure 5. Median magnetic field strength as a function of baryon density (top) and median gas temperature for the same gas density bins (bottom) for all models at $z=0.02$.

electrons may be accelerated in a two-phase fashion, in which they first gain energy via shock-drift acceleration if shocks are quasiperpendicular, and are later suitable for acceleration by DSA (e.g. Caprioli \& Spitkovsky 2014; Guo, Sironi \& Narayan 2014).

Following Banfi et al. (2020), we measured the shock obliquity from the shock propagation direction and the up-stream magnetic field $\boldsymbol{B}: \theta=\arccos \left(\frac{M_{x} \cdot B_{x}+M_{y} \cdot B_{y}+M_{z} \cdot B_{z}}{M B}\right)$ which ranges from $0^{\circ}$ to $180^{\circ}$. For the identification of up-stream magnetic field values and of the local shock propagation direction, we rely on our velocity-based shock finder (Banfi et al. 2020).

Fig. 7 shows the ratio between quasi-perpendicular $\left(|\theta| \geq 45^{\circ}\right)$ and quasi-parallel $\left(\theta<45^{\circ}\right)$ for all models as a function of the gas density, normalized to the ratio that is expected from a purely random distribution of vectors in 3D space (which follows a simple $\propto \sin \theta$ probability distribution).

All models present an excess of quasi-perpendicular shocks, with respect to a random distribution, in the $\rho \approx 10^{-32}-10^{-29} \mathrm{~g} \mathrm{~cm}^{-3}$ density range. The excess is maximal at the overdensity typical of filaments, which is explained by the fact that filaments tend to stretch the local magnetic field perpendicular to the velocity shear and to the local density gradient (Soler \& Hennebelle 2017), while shocks mostly run along the density gradient (Banfi et al. 2020). Therefore, in all models cosmic filaments are found in an environment in which the acceleration of CRs mostly proceed via quasi-perpendicular shocks, which would correspond to a reduced injection of CR protons in such structures (see Banfi et al. 2020; Wittor et al. 2020, for recent detailed analysis).

However, quantitative differences in the excess of quasiperpendicular shocks can be seen, with the highest excess being present in the uniform B0 model. This can be understood in terms of magnetic tension, i.e. of the difficulty by the gas flow in bending magnetic field lines. The tension increases with the field curvature, hence it is not surprising that in the B5 model, where the tangling of magnetic field lines is maximal at small scales since the beginning, the gas dynamics struggle more than in the B0 case to bend the field direction and to align it perpendicular to the gas density gradient. Intermediate trends are found in the other models. As an effect of small-scale dynamo in haloes, such effects are erased for $\rho \geq 10^{-28} \mathrm{~g} \mathrm{~cm}^{-3}$, and the distribution of shock angles become purely random (e.g. Wittor et al. 2017a).

Next, we measure the global volumetric and topological properties of magnetic fields for different epochs through their probability distribution functions (PDF) and their 3D power spectra, as shown by Figs 8 and 9, where we considered three evolutionary steps as an example: $z=5,0.4$, and 0.02 .

In the PDF, large differences are seen at all epochs, with the tendency to similarity of all models in the high magnetization regime $(\geq 0.1 \mu \mathrm{G})$ and for $z \leq 1$, after the small-scale dynamo has started operating in most haloes. However, for the bulk of the volume of the cosmic web the relative differences in the peak of the PDF are preserved from $z=5.0$ to $z=0.02$, and they mirror the differences at $z=40$, suggesting that in all cases the overall evolution of the PDFs is mostly driven by compression-rarefaction. This further supports that, at least in theory, it should be possible to constrain the magnetization model of our Universe, provided that we can probe the volumetric distribution of extragalactic magnetic fields today.

We computed the power spectra with standard Fast Fourier Transform techniques on the 3D grid, assuming periodicity, and in the case of the velocity spectrum, $P_{v}(k)$, we weighted the velocity variable by the square root of gas density, $v^{\prime}=\rho^{1 / 2} v$, so that the kinetic and magnetic spectrum, $P_{B}(k)$, have the same units. $P_{v}(k)$ is indistinguishable in the six runs, indicating that differences in the magnetic field at the level explored in this work cannot affect the overall gas kinematics in any important way. On the other hand, the evolution of $P_{B}(k)$ shows substantial differences on all scales for $z \geq 1.0$, while at lower redshift the spectra overlaps on scales $\leq 10-20 \mathrm{Mpc}$, as a result of the dynamo amplification. The similarity between models at small spatial scales is driven by our subgrid dynamo model. Since the small-scale dynamo erases any topological memory of initial fields in the real Universe (e.g. Cho 2014; Marinacci et al. 2015; Vazza et al. 2018), all models look alike. Already from this test, it is clear that the differences between models at low redshift will be minute and difficult to observe, because most of the difference is contributed by very large spatial scales, $\gg 10 \mathrm{Mpc}$, which are characterized by low densities and large angular scales in the sky.

\subsubsection{Properties of galaxy clusters}

We first examine the properties of our simulated population of galaxy clusters and groups, in order to check whether significant differences between extreme variations in the primordial seed fields can already be detected.

The typical difference between the magnetic field topology within and around the most massive halo for all investigated models is shown in Fig. 10. 

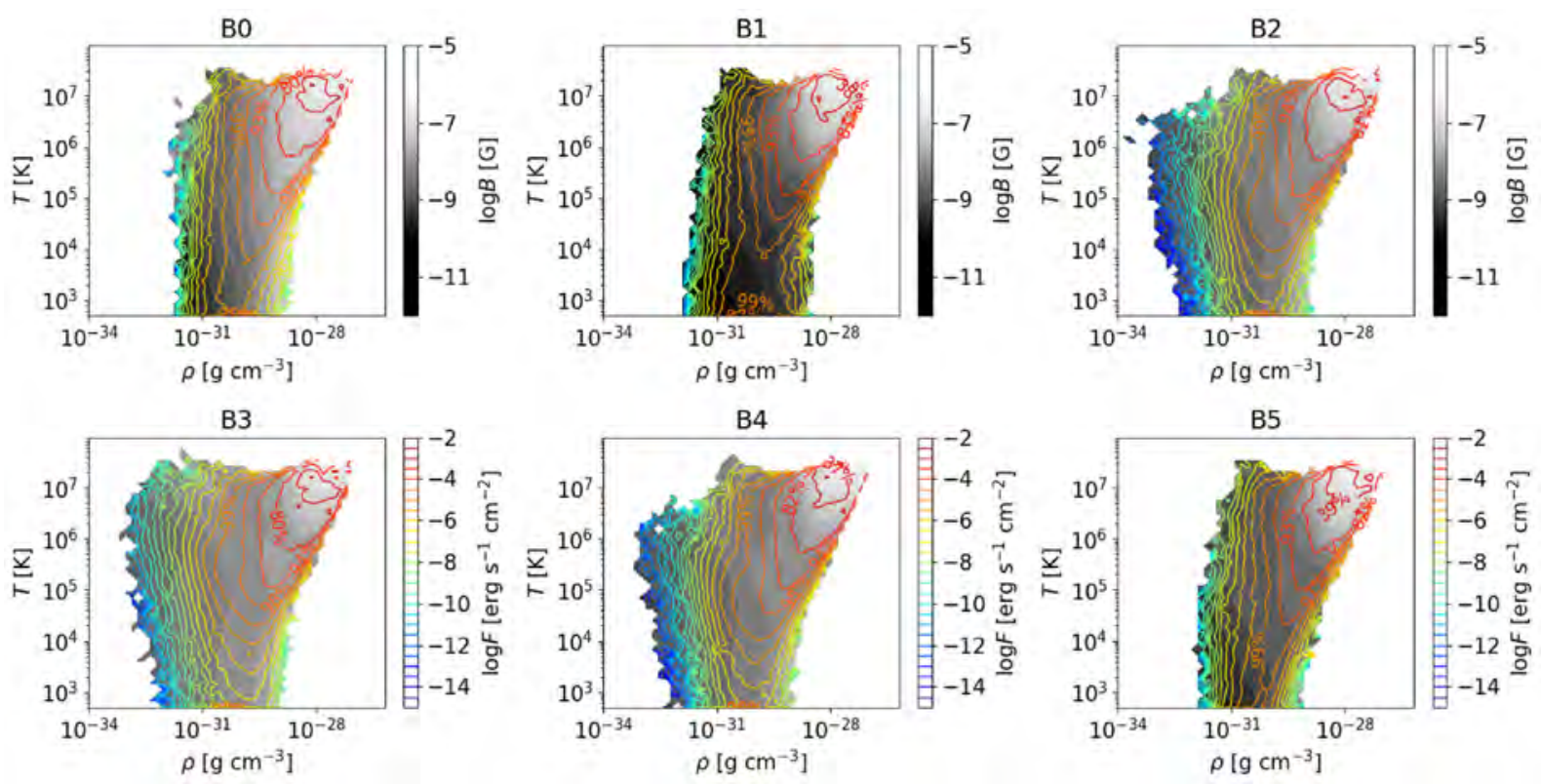

Figure 6. Phase diagrams of baryons in all simulations at $z=0.02$. The additional colour coding gives the median magnetic field strength in each gas phase (BW colour palette) and the total dissipated energy flux at shocks (contours).

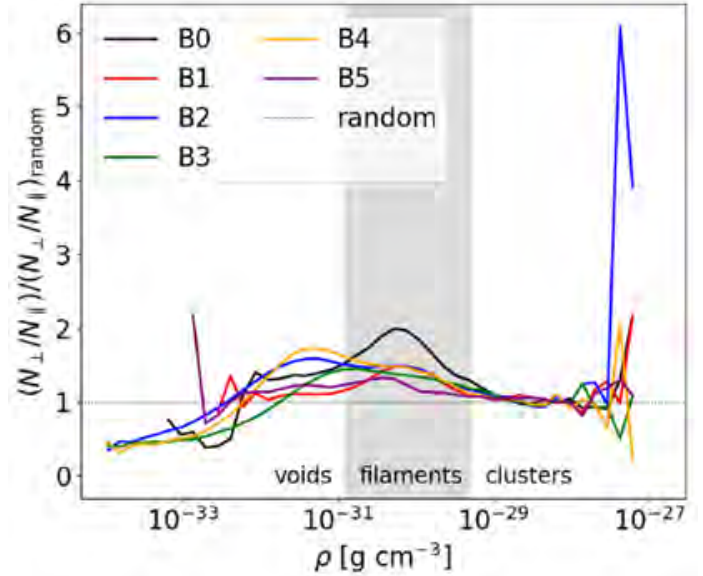

Figure 7. Distribution of the ratio quasi-perpendicular to quasi-parallel shocks, normalized to the expected ratio in a random $3 \mathrm{D}$ distribution, a function of gas density at $z=0.02$.

We identified all massive self-gravitating haloes with a standard analysis of the spherical (gas + DM) overdensity (e.g. Gheller, Pantano \& Moscardini 1998), and extracted the total mass and average (volume weighted) magnetic field strength within the radius enclosing a matter density 200 times the cosmological critical density $\left(R_{200}\right)$.

In principle, the presence of a large magnetic tension in the cosmological initial conditions may induce a change in the late evolution of the clustering properties of galaxy clusters, of their mass function as well as of their thermal scaling relations (e.g. Dolag et al. 1999; Dolag, Evrard \& Bartelmann 2001; Kahniashvili et al. 2013; Sanati et al. 2020). However, the top panel of Fig. 11 shows that the mass function of haloes identified in all simulations at $z=$ 0.02 are basically indistinguishable, across more than two orders of magnitude in mass, down to $\approx 10^{12} \mathrm{M}_{\odot}$. Moreover, the scaling relations between the cluster mass and the average gas temperature within $R_{200}$ show no differences (not shown).

On the other hand, larger differences are measured for the average (volume weighted) magnetic field strength within $R_{200},\left\langle B_{200}\right\rangle$, between the same objects. The best-fitting relations suggest that the differences are on average of order $\leq 10-20$ per cent between extreme models. This makes the detection of any difference on these small scales extremely challenging, because in observations the global cluster magnetic field can be either estimated through the (sparse) sampling of Faraday rotation from background polarized sources, and/or from the modelling of diffuse radio emission originating from shocks or turbulence (e.g. Brunetti \& Jones 2014; van Weeren et al. 2019). Therefore, we consider any differences of the magnetic field distribution inside clusters or groups of galaxies likely too weak to be detectable by observations.

Unlike in Kahniashvili et al. (2013), we consider only the stochastic background of primordial magnetic fields and do not account for the effect of magnetized perturbations on the matter power spectrum at small scales. For this reason, the effects of different models on the final distribution of cluster masses might be underestimated. However, even in the more self-consistent (analytical) work by Kahniashvili et al. (2013), significant effects of primordial magnetic fields on the halo mass function are confined to $\leq 10^{12} \mathrm{M}_{\odot}$ haloes for most models, i.e. in a range of masses which cannot be properly resolved by our runs here. In particular, Kahniashvili et al. (2013) reported that the number of small mass objects $\left(M \sim 10^{4} \mathrm{M}_{\odot}\right)$ in the most magnetized cases can be reduced by a factor $\sim 10^{2}$ compared to the unmagnetized case number, while an excess in the number haloes with respect to the unmagnetized case is found for objects with masses of $\sim 10^{10} \mathrm{M}_{\odot}$.

\subsection{Observable properties}

We compute the Faraday Rotation Measure (RM) across the full length of the simulated box, by integrating for each 1D beam of cells 

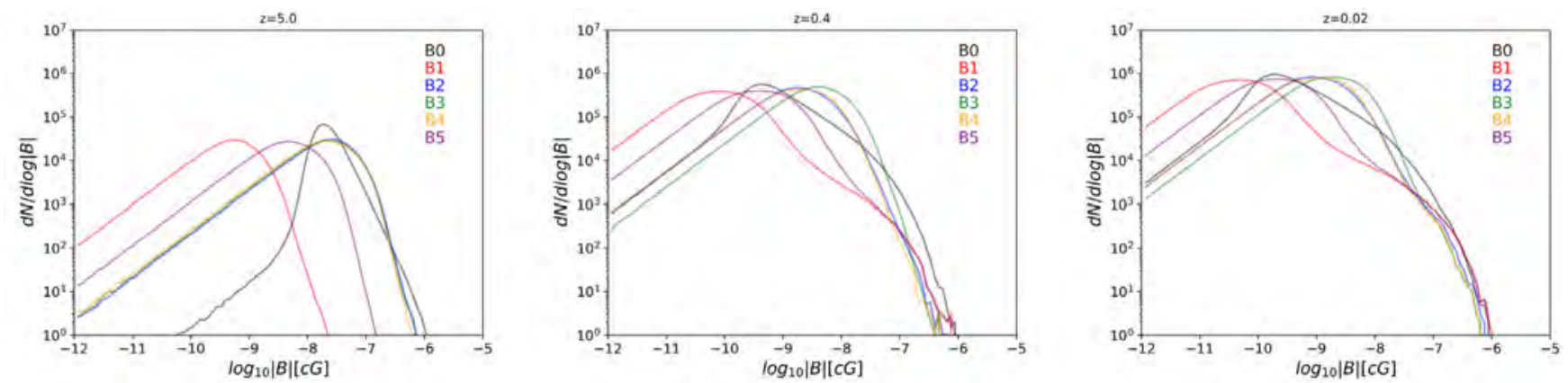

Figure 8. Probability distribution functions of comoving magnetic field strength for our models at four different epochs.
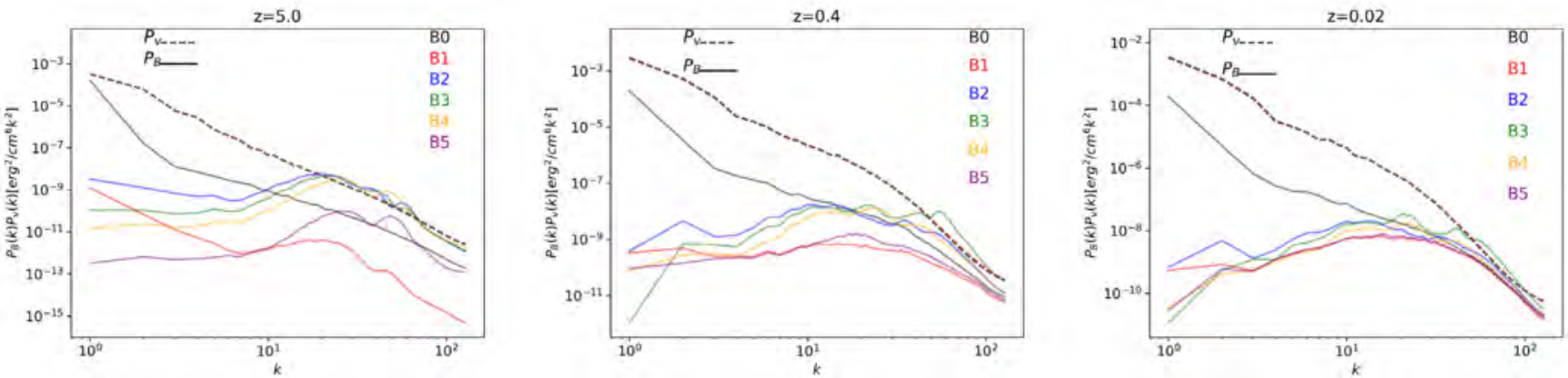

Figure 9. 3D magnetic power spectra (solid lines) and kinetic energy spectra (dashed lines) for our models at four different epochs.

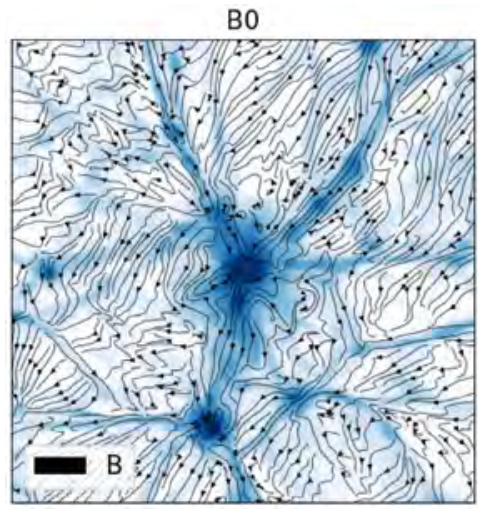

B3

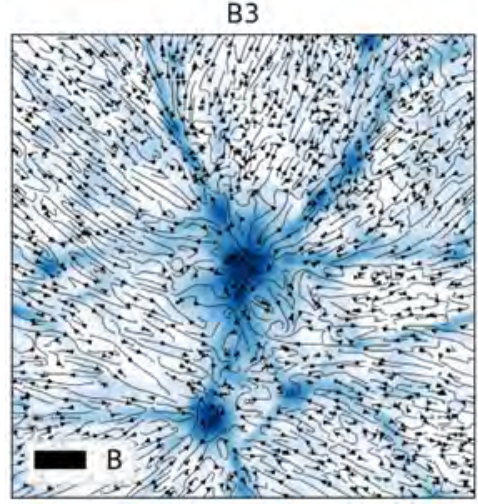

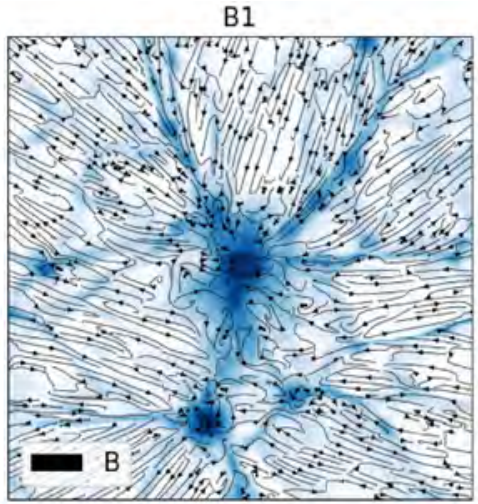

B4

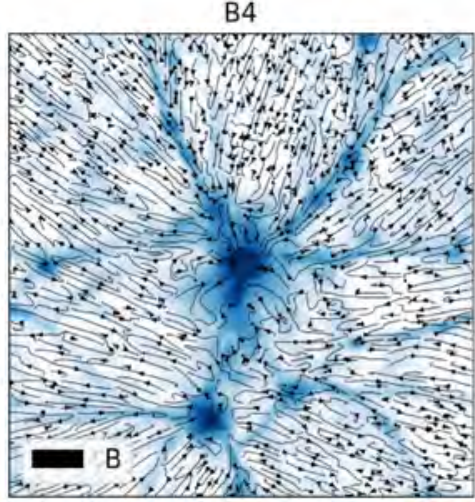

B2

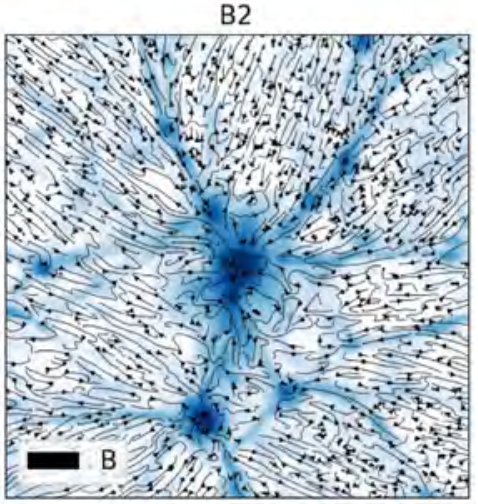

B5

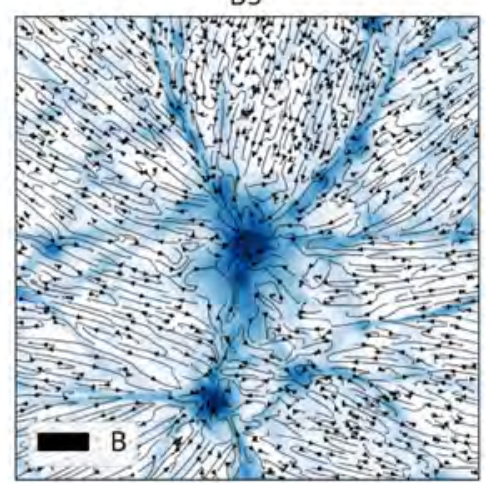

Figure 10. Thin slices (195 kpc thick) showing the gas density (blue) and the magnetic field lines (black) for a $20 \times 20 \mathrm{Mpc}^{2} \mathrm{zoom}$ around the most massive cluster in our simulated volume at $z=0$. 

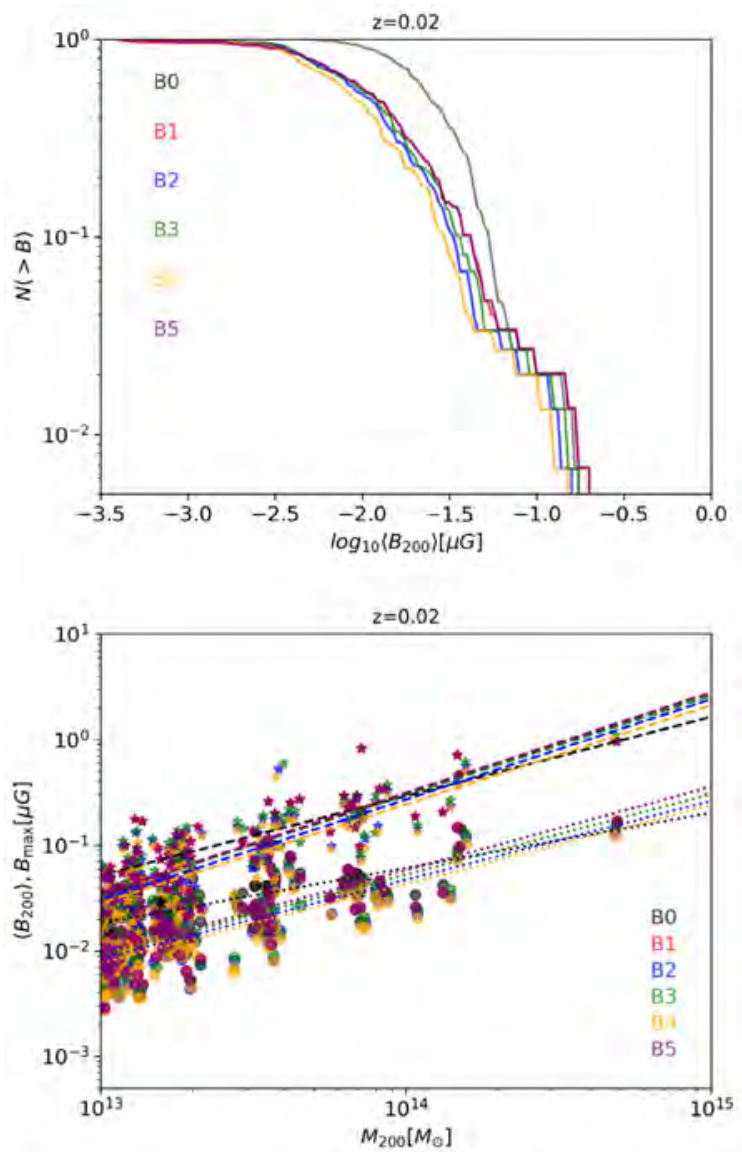

Figure 11. Top: cumulative mean magnetic field strength (volume averaged within $R_{200}$ ) for the same haloes. Bottom: scatter plot relating $M_{200}$ and the average $\left(\left\langle B_{200}\right\rangle\right)$ or the maximum $\left(B_{\max }\right)$ magnetic fields for the same clusters. The additional dashed/dotted lines gives the best fits of the two relations.

the quantity

$$
\frac{\mathrm{R}}{\left[\mathrm{rad} \mathrm{m} \mathrm{m}^{-2}\right]}=812 \int \frac{B_{\|}}{[\mu \mathrm{G}]} \cdot \frac{n_{\mathrm{e}}}{\left[\mathrm{cm}^{-3}\right]} \frac{\mathrm{d} l}{[\mathrm{kpc}]} \frac{1}{(1+z)^{2}},
$$

in which $B_{\|}$denotes the component of the magnetic field parallel to the line of sight (LOS), $z$ is the redshift of each cell, $n_{\mathrm{e}}$ is the physical electron density of cells, assuming a primordial chemical composition of gas matter everywhere in the volume $(\mu=0.59)$. RM thus corresponds to the Faraday rotation experienced by sources of linearly polarized radio emission, located in the background of our LOS.

To compute the diffuse synchrotron radio emission from the cosmic web, we limit here to the contribution from cosmic shocks (e.g. Vazza et al. 2015) and assume that relativistic electrons get accelerated by DSA. We closely follow the formalism by Hoeft \& Brüggen (2007), and compute the synchrotron emission for each shocked cell (identified with a velocity-based approach as in Vazza et al. 2009) as the convolution of the several power-law distributions of electrons that overlap in the cooling region downstream of each shock

$$
\begin{aligned}
\frac{\mathrm{P}(v)}{\left[\operatorname{erg~s}^{-1} \mathrm{~Hz}^{-1}\right]}= & 6.4 \times 10^{34} \cdot \int \frac{\xi_{e}(\mathcal{M}) \cdot A}{\mathrm{Mpc}^{2}} \frac{n_{\mathrm{e}}}{10^{-4} \mathrm{~cm}^{-3}}\left(\frac{T}{7 \mathrm{keV}}\right)^{3 / 2} \\
& \times\left(\frac{v}{\mathrm{GHz}}\right)^{-s / 2} \cdot \frac{B^{1+s / 2}}{B_{\mathrm{CMB}}^{2}+B^{2}} \frac{1}{(1+z)^{2}} \mathrm{~d} V
\end{aligned}
$$

in which $A$ is the shock surface, $T$ is the cell temperature, $s$ is the energy spectrum of accelerated particles, defined as $s=2\left(\mathcal{M}^{2}+\right.$ 1) $/\left(\mathcal{M}^{2}-1\right), B$ is the magnetic field (in $\mu \mathrm{G}$ and $B_{\mathrm{CMB}}$ is the CMBequivalent magnetic field. $v$ is the observing frequency, $\mathrm{d} V$ is the cell volume and $\xi_{e}(\mathcal{M})$ is the acceleration efficiency of electrons, which is $\leq 10^{-4}$ for weak shocks $(\mathcal{M} \leq 3-4)$ and it reaches $\sim 10^{-2}$ for strong, $\mathcal{M} \gg 10$ shocks.

We give an example of the distribution of synchrotron radio emission (with the projected DM density overlaid) emitted from the shocked cosmic web for all our models in Fig. 12.

The panels in Fig. 13 give the distribution of RM and of radio power for all galaxy clusters in our volume, limited to their peripheral $\geq \mathrm{R}_{200}$ regions, which are the typical location of peripheral radio relics (van Weeren et al. 2019).

The best-fitting relations for the relation between the radio emission or the Faraday rotation and $M_{200}$ of haloes have quite similar slopes, while their differences in normalization follow from the different trends in the average magnetic field found in Fig. 11. Interestingly, this suggests that already outside of $R_{200}$ there is a residual difference in radio observables, that is not entirely erased by the action of the small-scale dynamo amplification. A higher value of RM and synchrotron emission is expected for the simple B0 model, consistent with the systematically higher average fields found in the previous section. However, the scatter present in both quantities within all mass bins is much larger than the difference between models. Because of this, only with very large surveys of clusters it might be possible to reduce the effect of cosmic variance, which are instead a big limitation with samples of $\leq 50$ objects as the one simulated here.

\subsubsection{Cosmological light cones}

In the remainder of the paper, we consider the integrated synchrotron radio emission or Rotation Measure from light cones which are longer than our simulated volume, in order to produce a more realistic match with what observations can do. In detail, we used stacked 10 different redshift snapshots for each of our model resimulations, by extracting a corresponding cone within a fixed aperture $\left(\theta=2^{\circ}\right)$. The light cone was build by adding the information within each volume at regular intervals of $100 \mathrm{Mpc}$ (comoving) along the line of sight, and by introducing random shifts across the plane of the sky to avoid repeating patterns, until producing a $0.02 \leq z \leq 0.8$ light cone. An example of the integrated Faraday rotation for a light cone in the three reference models B0, B1, and B4 is shown in Fig. 14.

We show the resulting distributions of synchrotron radio emission (at $v=1.4 \mathrm{GHz}$ ), and of Faraday rotation for background polarized sources in Fig. 15. We additionally mark the regions traced by 'filaments' based on the projected mass-weighted gas temperature, using a fiducial threshold of $T_{\text {fila }}=10^{7} \mathrm{~K}$ to disentangle the signal produced by haloes from the one produced by more rarefied regions.

Small, but non-negligible differences, appear between different models, albeit in a regime which is challenging to observe with current instruments. There is a level of difference from filaments in the cosmic web (dotted lines), which seems to be at the edge of detectability both in Faraday rotation and in synchrotron emission. It is also worth noticing that there is a systematic difference in Faraday rotation between a simplistic uniform primordial magnetic field model (B0) and all other models. The B0 model yields an 3-5 higher Faraday rotation for filaments, compared to all other models, consistent with our previous results on clusters, and following from the fact that the rather laminar magnetic field lines in the bulk 


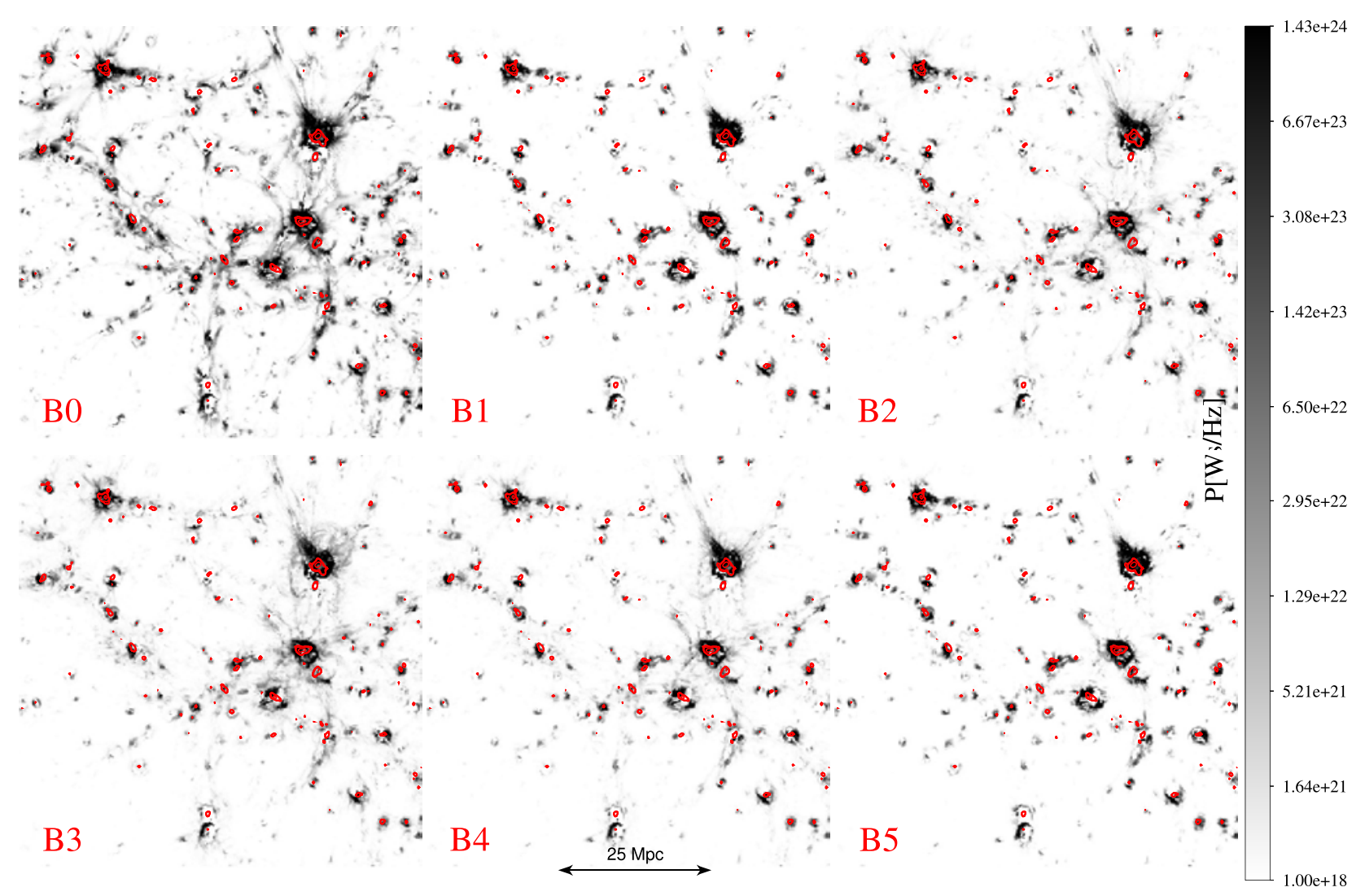

Figure 12. Synchrotron radio emission $(v=100 \mathrm{MHz})$ from cosmic shocks along the entire $100 \mathrm{Mpc}$, and projected DM density (red contours) for all our models at $z=0$.

of cosmic volume do produce a systematic Faraday rotation of background sources.

\subsubsection{Comparison to available radio observables}

A few authors have claimed a derivation of upper limits on the average magnetization of the cosmic web, based on the statistical analysis of radio surveys (e.g. Vernstrom et al. 2017, 2019; O'Sullivan et al. 2020). Our simulations can already be used for a first tentative comparison with recent work, albeit within the caveat posed by their limited resolution, which are discussed in detail in Section 4 and in the Appendix.

First, we attempt a qualitative comparison with the recent results by Vernstrom et al. (2017), who have cross-correlated the MWA Phase I radio observations and the large-scale distribution of galaxies observed with the WISE+2MASS galaxy survey, for a $22^{\circ} \times 22^{\circ}$ field of view. Vernstrom et al. (2017) reported no significant detection of cross-correlation between galaxies and radio emission on $\geq 20$ arcmin scales, and used this information to derive upper limits on the typical magnetization of filaments of the cosmic web. In Gheller \& Vazza (2020), we introduced a procedure to simulate the crosscorrelation between the diffuse radio emission at $180 \mathrm{MHz}$ and the projected galaxy distribution in the simulation. In particular, we set the threshold of projected DM density to $\rho_{\text {th }}=6 \times 10^{-29} \mathrm{~g} \mathrm{~cm}^{-3}$ in order to produce a number of galaxies (i.e. DM haloes) compatible with the sensitivity of the WISE IR survey, yielding $\sim 10$ galaxies per square degree for $z \leq 0.07$ (Vernstrom et al. 2017). For the radio emission, we considered the MWA Phase I sensitivity and resolution beam as in Vernstrom et al. (2017), by convolving our radio sky model for a $\theta \approx 2.9$ arcmin resolution beam and considering a (spatially uniform) noise level of $0.96 \mathrm{mJy}$ beam $\approx 0.028 \mu \mathrm{Jy} \operatorname{arcsec}^{-2}$ at $180 \mathrm{MHz}$.

Following Gheller \& Vazza (2020), the normalized correlation matrix $C$ between 2D $N \times M$ pixels images $A$ and $B$ is evaluated through

$C(k, l)=\frac{1}{N M} \sum_{j=0}^{N-1} \sum_{i=0}^{M-1} \frac{(A(i, j)-\bar{A})(B(i+k, j+l)-\bar{B})}{\sigma_{A} \sigma_{B}}$,

where $\bar{A}$ and $\bar{B}$ are the mean values of the two images and $\sigma_{A}$ and $\sigma_{B}$ are their standard deviation, while the indices of the correlation matrix $C$ give the shift (displacement) of the two images. The significance of the cross-correlation is evaluated against the case of null correlation, for which $C$ is computed between $A$ and $B$ from uncorrelated $50 \times 50 \mathrm{Mpc}^{2}$ sub-tiles extracted from the main simulation.

The results are shown in Fig. 16; they cannot be readily compared with the cross-correlation values given by Vernstrom et al. (2017), owing to the different approaches in estimating the noise level of the cross-correlations. Therefore, our synthetic observation can only qualitatively address which model seems to be more compatible with MWA observations. While all models give a significant crosscorrelation out to $\sim 40-50$ arcsec, the amplitude of the correlation in the B1, B4, and B5 models (and, to a smaller extent, over the homogeneous B0 model) seems to be too large to have been missed by MWA observations. This potentially suggests already that a $1 \mathrm{nG}$ initial field (resulting into a typical magnetization of filaments of 

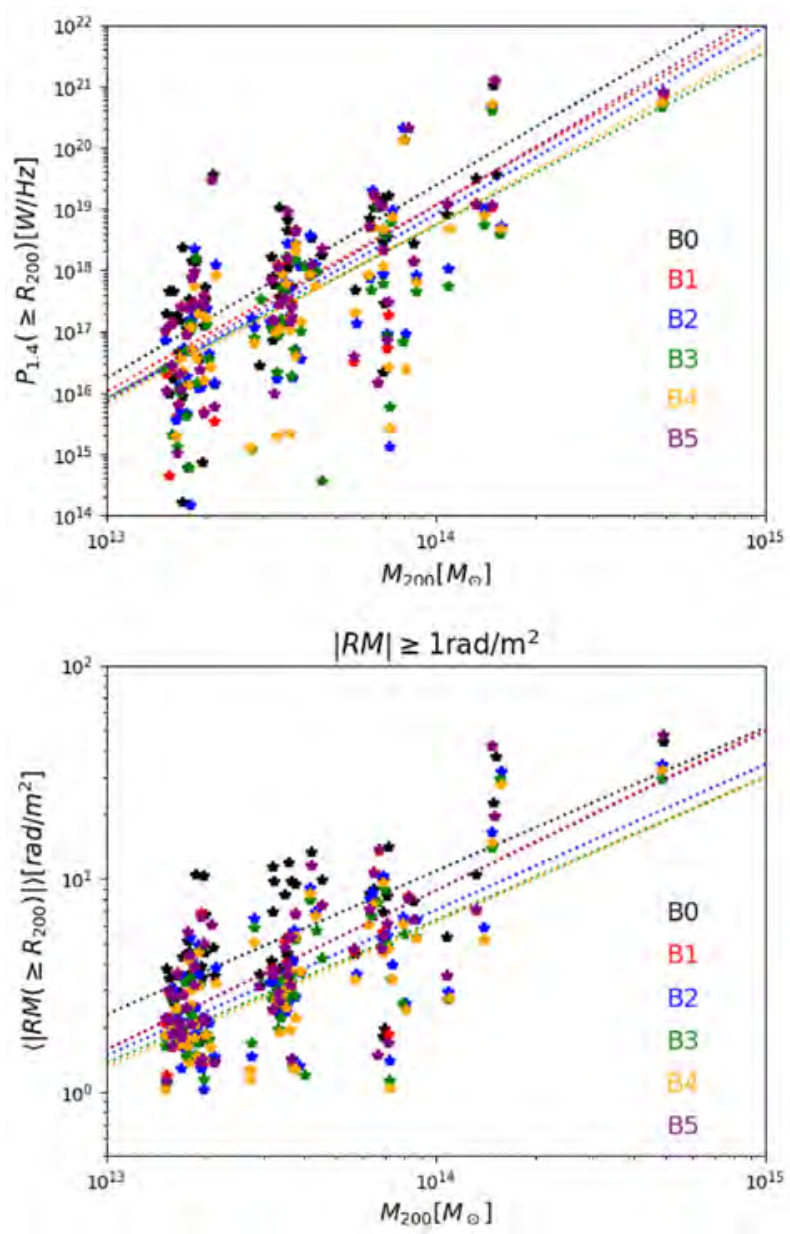

Figure 13. Top: radio power from shocks at $1.4 \mathrm{GHz}$ from peripheral, $R_{200} \leq$ $r \leq 2 R_{200}$ regions versus $M_{200}$ at $z=0.02$ for the same clusters in all runs. The dotted lines show the best-fitting relations for each model. Bottom: average $|\mathrm{RM}|$ in the range $R_{200} \leq r \leq 2 R_{200}$, considering a (future) $|\mathrm{RM}| \geq 1 \mathrm{rad} \mathrm{m}^{-2}$ sensitivity. The dotted lines show the best fit relations for each model.

10-100 nG as shown in Gheller \& Vazza 2019) is too large to be compatible with the lack of detection reported by Vernstrom et al. (2017). Interestingly, intermediate models B2 and B3 present crosscorrelation only at the $\leq 2 \sigma$ level and on slightly smaller angular scales.

As a caveat, given the finite mass resolution of our simulations, we cannot properly form dwarf galaxies in voids (or in very poor environment, in general). Therefore, even if the number of galaxies is calibrated to be at the level of the galaxy distribution observed in WISE/MASS surveys, our spatial distribution is typically more clustered than in observations. In principle, this can decrease the cross-correlated signal coming from low-density regions in our sample. With future work, we will employ more resolved simulations in order to address this issue.

O'Sullivan et al. (2020) recently reported limits on the Faraday depth contribution of the magnetized cosmic web, by comparing the structure function of Faraday rotation in random pairs of radio galaxies compared to physical radio galaxies (i.e. doubled-lobed radio galaxies), on overlapping angular scales.

We produced mock structure functions of Faraday rotation for our simulated deep light cones, following the same procedure as in O'Sullivan et al. (2020). We integrated the RM along the line of sight down to $z=0.7$, and computed the statistic of $\left\langle(\Delta R M)^{2}\right\rangle$ for physical pairs of pixels, by placing pairs of sources at regular intervals of $100 \mathrm{Mpc}$ (comoving) along the line of sight (i.e. at the end of each of the comoving volumes used to produce the stacking sequence of Faraday rotation). In detail, we first randomly drew 20000 sources (with $|\mathrm{RM}| \geq 0.03 \mathrm{rad} \mathrm{m}^{-2}$ as in the LOFAR observation, for 10 evenly spaced redshift bins, and calculated $\left(\Delta R M(\Delta \theta)^{2}\right)$ at each redshift. The assumed angular resolution for the light cone of Faraday rotation is the same of O'Sullivan et al. (2020) LOFAR-HBA observation, i.e. $\approx 20$ arcsec. The final distribution of $\left(\Delta R M(\Delta \theta)^{2}\right)$ can be compared with recent LOFAR-HBA observations by O'Sullivan et al. (2020), by weighting each structure function in redshift bins for the (likely) redshift distribution function of sources, derived in Vernstrom et al. (2019).

Fig. 17 gives the simulated distribution of $\left(\Delta R M(\Delta \theta)^{2}\right)$ as a function of angular separation for all our models, compared with real LOFAR-HBA data (grey area). At face values, the structure functions of models B1 and B5 appear strongly disfavoured by the comparison with observations, while all other models remain compatible with LOFAR data, even if it is non-trivial to evaluate the uncertainties in the signal from simulations, owing to the limited volume scanned here as well as to the different selection function of sources in redshift applied to simulated data. The trend for B1 and B5 is in striking good agreement with the previous test on the cross-correlation of synchrotron emission and the galaxy distribution, in the sense that these two models already likely to ruled out by radio observations. However, our limited spatial resolution is likely to underestimate the Faraday structure below $\leq 200 \mathrm{kpc}$, with a stronger effect than in the case of synchrotron emission, because Faraday rotation is more sensitive to the (unresolved) small-scale topology of magnetic and density perturbations. We comment on this issue more in detail in Section 4 and in the Appendix. Moreover, it is important to notice that the above structure functions still have an unknown contribution from the Rotation measure variance of the Milky Way. In case this is dominant on $\leq 10$ arcmin scales, the observational constraints from $\left(\Delta R M(\Delta \theta)^{2}\right)$ will decrease to lower values, possibly putting tension on all models investigated here.

\subsubsection{The deflection of ultra-high-energy cosmic rays}

Finally, we investigated whether the propagation of ultra-highenergy cosmic rays across cosmic distances is differently affected by our different magnetic field models. Cosmic rays with energies $\geq 10^{18} \mathrm{EeV}$ are indeed believed to have a predominantly extragalactic origin, and their propagation towards Earth must be deflected by the Lorentz force by extragalactic magnetic fields (e.g. Sigl, Miniati \& Ensslin 2003; Dolag et al. 2005). Large deflections hamper the possibility of locating the real sources of UHECRs at the highest energies (e.g. Biermann et al. 2016), yet the amplitude of these deflections can greatly vary depending on model assumptions, ranging from $\leq 1^{\circ}$ to $\sim 30^{\circ}$ (e.g. Dolag et al. 2005; Alves Batista \& Sigl 2014; Berezinsky 2014;). We recently studied the propagation of UHECRs in ENZO simulations combined to the CRPROPA code (Alves Batista et al. 2016), finding that the observed level of isotropy of $\geq 10^{18} \mathrm{eV}$ UHECRs rules out primordial models with a uniform magnetic field larger than $\sim 10 \mathrm{nG}$ (Hackstein et al. 2016, 2019).

The typical deflection angle, $\theta$, of a cosmic ray of energy $E$ and charge $Z$ propagating through the extragalactic magnetic field can be simply estimated as

$\theta(E)=0.8 \mathrm{Z}\left(\frac{\mathrm{E}}{10^{20} \mathrm{eV}}\right)^{-1} \cdot\left(\frac{\mathrm{r}}{10 \mathrm{Mpc}}\right)^{1 / 2} \cdot\left(\frac{\lambda_{\mathrm{B}}}{\mathrm{Mpc}}\right)^{1 / 2} \cdot \frac{\mathrm{B}}{\mathrm{nG}}$ 


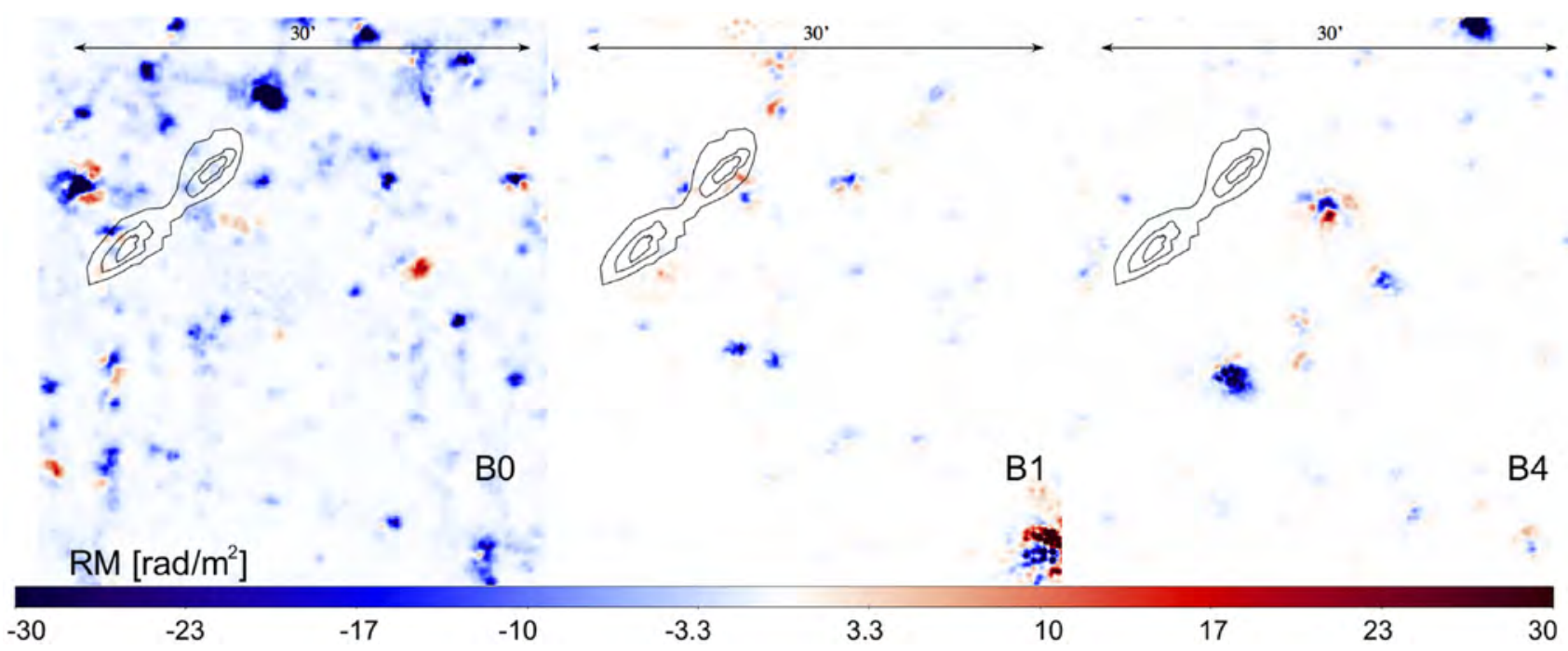

Figure 14. Integrated Faraday rotation for an $\sim 30 \operatorname{arcmin} \times 50$ arcmin field of view with depth until $z=0.5$, for models B0, B1, and B4. The maps are convolved for a 20 arcsec resolution beam. The simple sketch of an FRII radio galaxy is added to refer to our analysis discussed in Section 3.2.1.
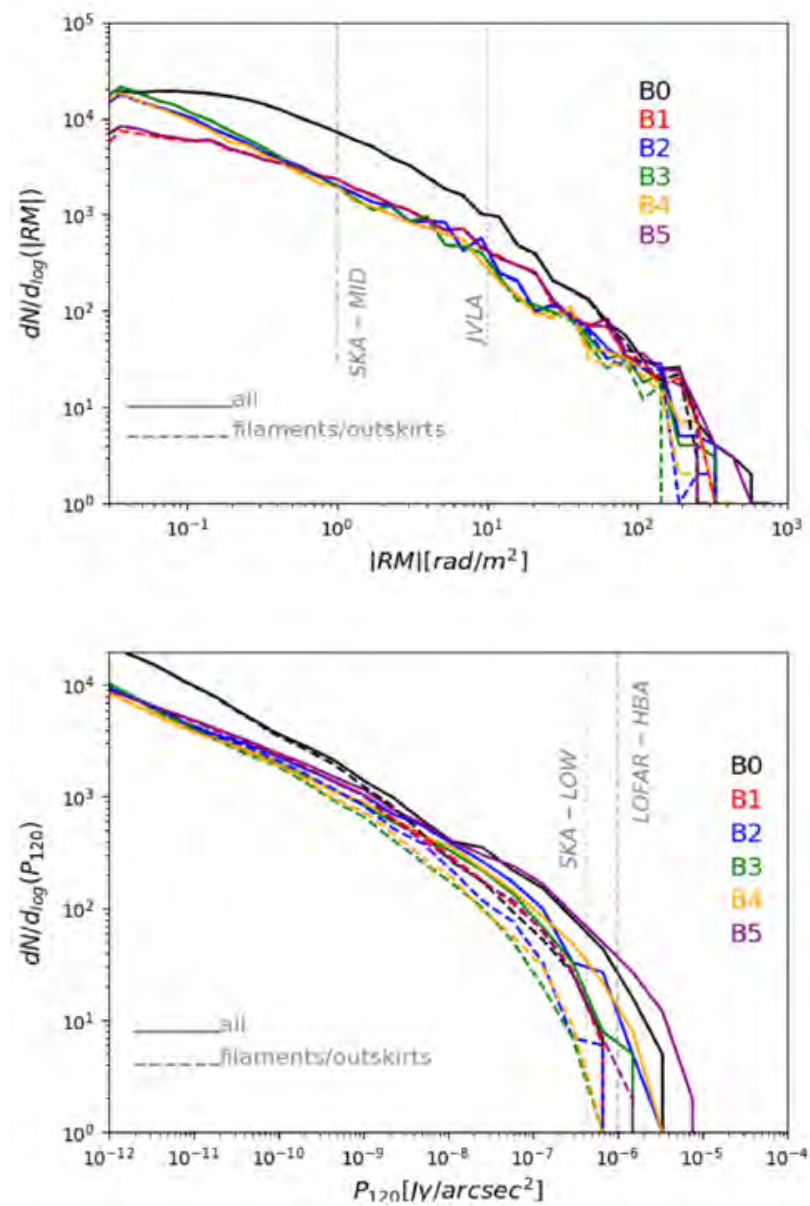

Figure 15. Top: distribution functions of Faraday rotation for all models and a $2^{\circ} \times 2^{\circ}$ field of view up to $z=0.8$. Bottom: distribution function of synchrotron radio emission at $1.4 \mathrm{GHz}$ for all models and a $2^{\circ} \times 2^{\circ}$ field of view up to $z=0.8$.

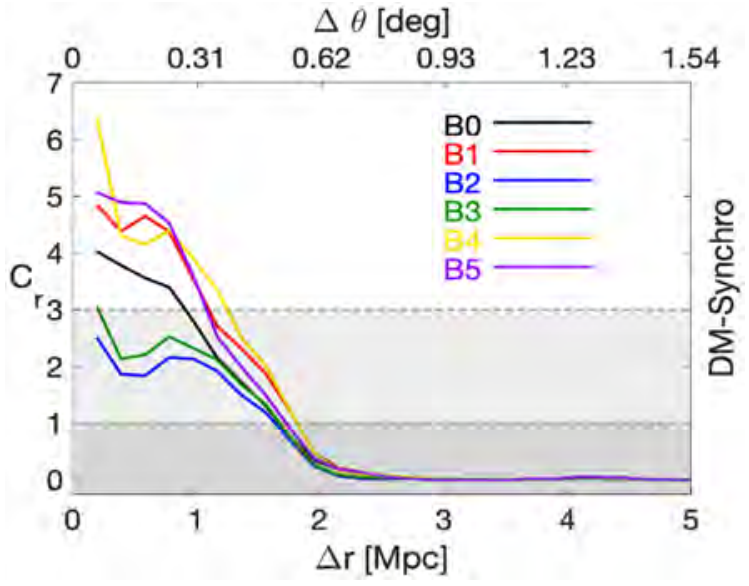

Figure 16. Simulated cross-correlation between the projected DM distribution (simulating galaxies) and the synchrotron emission detectable by MWA, using the observing configuration by Vernstrom et al. (2019). The crosscorrelation is normalized to the corresponding null model and the horizontal lines mark the $1 \sigma$ and $3 \sigma$ detection level.

where $\lambda$ is the typical coherence length of magnetic fields (assumed in this case to be constant across the cosmic volume, and fixed to $\lambda_{B}=1 \mathrm{Mpc}$ here for simplicity), and $r$ is the propagation length (e.g. Sigl et al. 2003).

The maps in Fig. 18 give a visual impression of the average deflection angle computed from the above formula, for runs B0, $\mathrm{B} 1$, and $\mathrm{B} 4$ at $z=0.02$. Very large $\left(\theta \gg 10^{\circ}\right)$ deflection angles are expected for UHECR protons crossing the virial regions of haloes, due to the $\geq 0.1 \mu \mathrm{G}$ field developed there. In the case of the uniform B0 model; however, the deflection is significant in filaments too, and far from negligible for the entire projected sky $\left(\theta \sim 10^{\circ}\right)$. Deflections are still non-negligible in the $\mathrm{B} 5 \operatorname{model}\left(\theta \sim 2^{\circ}\right)$, while in the model B1 the deflection is almost negligible $\left(\theta \leq 1^{\circ}\right)$ for most of the projected area, once haloes are excluded.

A proper simulation of the effect of deflection by extragalactic magnetic fields requires to properly integrate the trajectories of cosmic rays crossing our simulated volume. We thus simulated the propagation of UHECRs by integrating the trajectories of 2000 


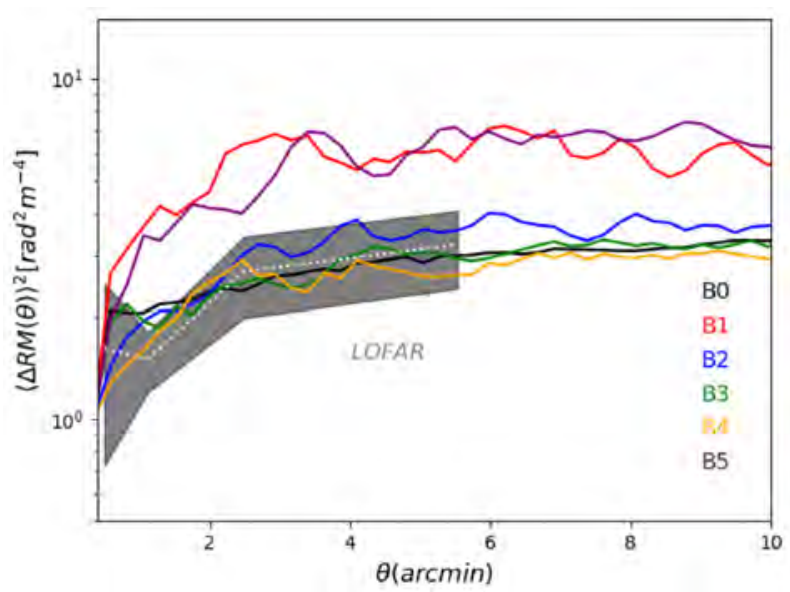

Figure 17. Simulated distribution of $\left(\Delta R M(\Delta \theta)^{2}\right)$ as a function of angular separation for all our models. The grey area shows the LOFAR $144 \mathrm{MHz}$ data by O'Sullivan et al. (2020), with noise power from measurement errors subtracted, for physical pairs of radio sources only.

randomly distributed cosmic rays for each model, assuming a random initial orientation of their velocity (which is of course $|v|=c$ ) and we integrated their propagation through the simulated $100^{3} \mathrm{Mpc}^{3}$ volume in all models. In detail, their propagation was integrated by computing the deflection by the Lorentz force with a kickdrift-kick second-order integration scheme (e.g. Ryu, Das \& Kang 2010), assuming that all cosmic rays are protons $(Z=1)$ with an initial energy of $10^{18}, 10^{19}$, or $10^{20} \mathrm{eV}$, and neglecting all other loses of energy via other processes, which is a reasonable enough assumptions considering the relatively small propagation length considered here. The maps in Fig. 19 show the paths of a sample of protons through runs B0, B1, and B5, while in Fig. 20 we give the distributions of deflection angles for the protons with the three possible initial energies, by computing $\theta$ as the angle between the initial injection direction and the final velocity vector, either after 30 or $100 \mathrm{Mpc}$ of propagation length since their origin. Consistent with the expectations of deflection angles, we observe a large difference in the typical propagation of UHECR protons in the B0, B1, and B5 case. The largest deflection is measured in the B0 scenario, in which the distribution of directions of UHECRs of $10^{20} \mathrm{eV}$ gets entirely randomized after propagating for $\sim 100 \mathrm{Mpc}$. For most of observers in this simulated Universe it will be thus entirely impossible to track the origin of received UHECRs, backwards to their original injection location. In most investigated models, already after $\sim 30 \mathrm{Mpc}$ of propagation the observed UHECR protons (for $E \leq 10^{20} \mathrm{eV}$ ) are so highly deflected, that that 'UHECRs astronomy' would impossible. Only in the $\mathrm{B} 1$ and in the $\mathrm{B} 5$ models $10^{20} \mathrm{eV}$ protons are not too much deflected after $30 \mathrm{Mpc}$ of propagation, and their deflection also peaks at small angles after $100 \mathrm{Mpc}$.
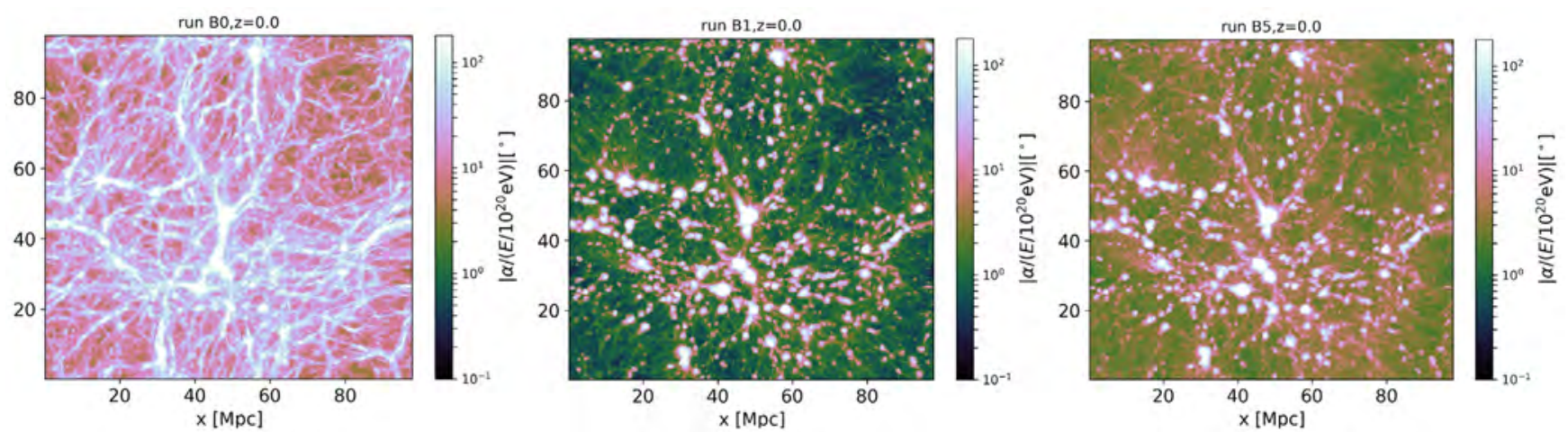

Figure 18. Deflection angle for $E=10^{20} \mathrm{eV}$ protons, averaged along a $100 \mathrm{Mpc}$ line of sight in models B0, B1, and B5.
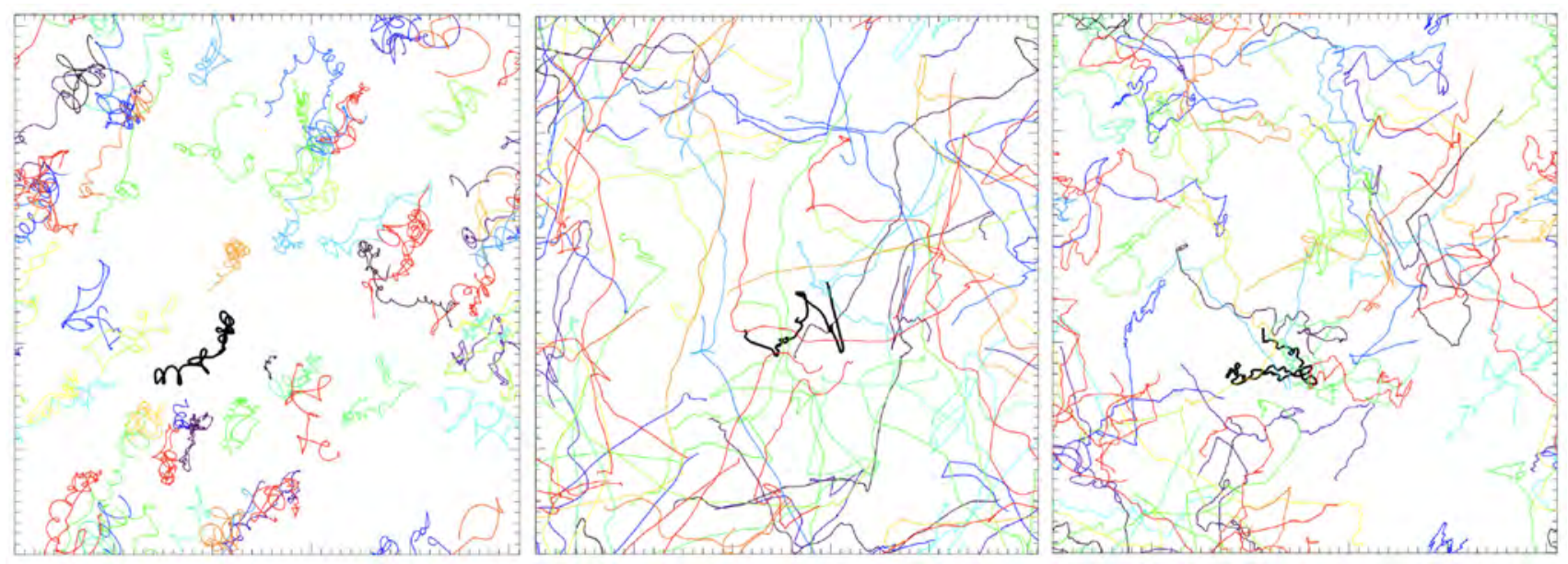

Figure 19. Trajectories of $E=10^{19} \mathrm{eV}$ protons crossing our entire $100^{3} \mathrm{Mpc}^{3}$ volume in models $\mathrm{B} 0, \mathrm{~B} 1$, and $\mathrm{B} 5$. Each cosmic ray has been randomly injected at the opposite side of the box with respect to the observer. 

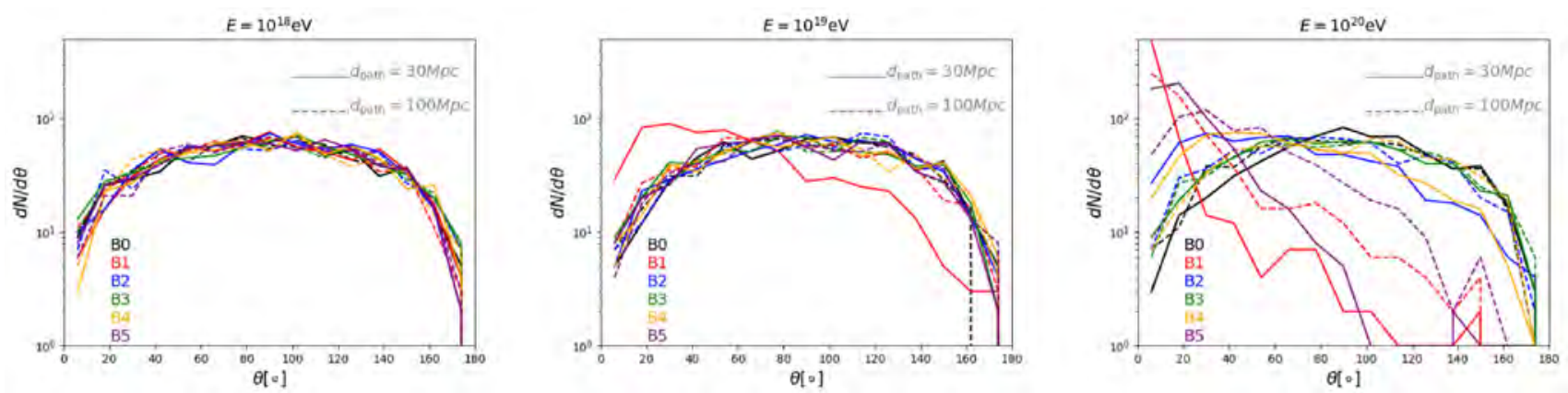

Figure 20. Distribution of deflection angles for simulated UHECR protons with an initial energy $E=10^{18} \mathrm{eV}$ (top) or $E=10^{19} \mathrm{eV}$ (centre) and $E=10^{20} \mathrm{eV}$ (bottom), injected with initial random positions and velocities in our simulated volumes. The different linestyles in both panels show the distribution of deflection angles after propagating for $30 \mathrm{Mpc}$ (solid) or $100 \mathrm{Mpc}$ (dashed).

This is consistent with previous results that investigated the propagation of UHECRs (also taking into account their energy losses) with ENZO simulation and a similarly large primordial field (Hackstein et al. 2016, 2019).

In summary, we find that among the investigated models there is the theoretical possibility of detecting signatures of the topology of seed magnetic fields in voids, through the clustering of $10^{20} \mathrm{eV}$ events from very nearby $\leq 10 \mathrm{Mpc}$ sources. Unluckily, the statistics of such events is presently so small that such analysis will be dominated by sample variance in the real case (Hackstein et al. 2019).

\section{DISCUSSION}

\subsection{Numerical and physical limitations}

A few important limitations to our work, connected to the employed numerical scheme, must be taken into account in the interpretation of our results.

Probably, the most important limitation of this set of simulation is the adopted spatial resolution, which combined with our sub-grid dynamo model is enough to follow the evolution of magnetic fields and shocks on $\geq 200 \mathrm{kpc}$ scales (as discussed in several previous work, e.g. Vazza, Gheller \& Brüggen 2014a; Vazza et al. 2014b), but likely not enough to simulate cell-by-cell fluctuations of RM, which are key to interpret the structure functions discussed in Section 3.2.2. We present in the Appendix the additional tests with a smaller series of runs, designed to test the dependence of our results on the adopted spatial resolution. As expected, the structure function for RM shows a significant, albeit non-dramatic, evolution with resolution. This presently makes it impossible to firmly establish which of our models for primordial magnetic fields can already be ruled out by comparison with the LOFAR data of O'Sullivan et al. (2020), and only future simulations at a higher resolution will be able to make a quantitative comparison.

We notice that RM fluctuations on scales smaller than the LOFAR synthesized beam ( 20 arcsec i.e.$<150 \mathrm{kpc}$ at $z=0.8$ ) would cause wavelength-dependent depolarization (e.g. Burn 1966). In this case, the emission from a population of sources that probe regions of high RM variance on small scales are likely to be missing from the LOFAR data at $144 \mathrm{MHz}$ (i.e. completely depolarized), but would be present in data at a higher frequency, such as at $1.4 \mathrm{GHz}$ in the NVSS (Vernstrom et al. 2019). Recently, a depolarization study of LOFAR polarized sources found that they have a typical RM dispersion within the beam of $<0.3 \mathrm{rad} \mathrm{m}^{-2}$ (Stuardi et al. 2020). Therefore, any radio galaxy that is embedded in an environment with RM fluctuations larger than this on scales smaller than 20 arcsec will not be present in the LOFAR data, but can be detected at $1.4 \mathrm{GHz}$ (for RM dispersions up to a few $10 \mathrm{~s}$ of $\mathrm{rad} \mathrm{m}^{-2}$ ). Thus, high-resolution simulations, that include radio galaxies embedded in realistic environments that have RM fluctuations on a wide range of scales, can help statistically isolate those sources whose RM variance is dominated by the local environment (e.g. the intracluster medium) and those that are more pristine probes of cosmic filaments.

In this work we were only concerned with ideal MHD, meaning that the resistive dissipation of magnetic fields, the onset of 'microscopic' plasma instabilities and other effects related to the departure from a single fluid model were not included (e.g. Schekochihin et al. 2005).

Finally, in order to solely focus on the possible differences between variation of primordial seeding models, we neglected alternative scenarios for the seeding of magnetic fields related with galactic activity (e.g. Kulsrud et al. 1997; Kronberg, Lesch \& Hopp 1999; Völk \& Atoyan 2000; Langer, Aghanim \& Puget 2005; Donnert et al. 2009; Bertone, Vogt \& Enßlin 2006; Samui, Subramanian \& Srianand 2017).

However, the most important differences between primordial models we find concern the very peripheral regions of galaxy clusters, or filaments and cosmic voids, in which the impact of 'astrophysical' sources of magnetization must be sub-dominant (e.g. Vazza et al. 2017a; Hackstein et al. 2019). Therefore, the main differences found in this work are very likely to persist regardless of other sources of magnetization in large scales, if primordial magnetic fields do exist.

\section{CONCLUSIONS}

We presented cosmological magnetohydrodynamical simulations for six different initializations of 'primordial' seed magnetic field. We adopted models designed to be compatible with the conservative upper limits of non-helical primordial magnetic fields derived from the latest Planck, BICEP/Keck Array, SPT CMB anisotropy power spectra data as constrained in Paoletti \& Finelli (2019). We investigated the impact of the scale dependence of primordial magnetic fields by assuming a stochastic background, spanning different possible field configurations. The scale dependence is related to the generation mechanism and advance in this topic could be important in the understanding of the physics of the early Universe. In particular, causal mechanisms, mainly related to first-order phase transitions and second-order perturbations, require a spectral index $\alpha \geq 2.0$ that represents the extreme blue limit of our analysis. Inflationary mechanism can instead generate fields with also 'red' spectra that 
we sample starting from the lowest infrared limit $\alpha=-2.9$. We have limited our analysis to the effect of primordial magnetic fields at the stochastic background level, leaving the study of a modified matter power spectrum due to magnetized cosmological perturbations for a future work.

We find that while matter haloes at low $-z$ do not retain memory of seed fields (due to the fact that their magnetic field is dominated by dynamo amplification), their outskirts, filaments, and voids might retain traces of the topology and amplitude of the primordial fields.

Our main findings can be so summarized as follows:

(i) different models of primordial seed fields, all designed to be consistent with the most recent conservative constraints from the combination of the recent CMB data of Planck 2018 and groundbased observatory BICEP/KECK and South Pole Telescope (Paoletti \& Finelli 2019), produce significant differences in the average strength and topology of magnetic fields in the less dense cosmic environment, i.e. for $\rho \leq 10^{-30} \mathrm{~g} \mathrm{~cm}^{-3}$, even down to $z=0$;

(ii) depending on the amplitude of magnetic field fluctuations at small scales $(\ll 10 \mathrm{Mpc}$ ) additional gas perturbations, eventually leading to shock waves, are driven in the most rarefied cosmic gas, in addition to 'standard' structure formation shocks (albeit adding little extra energy in the total volume);

(iii) all variations of the magnetic field scenarios have no relevant impact on the formation of structures, at least for the $\geq 10^{12} \mathrm{M}_{\odot}$ masses probed in this work;

(iv) the investigated properties of magnetic fields in galaxy clusters, and of the observables connected to them, are almost invariant within $\leq \mathrm{R}_{200}$ of simulated clusters. Differences between models, which can be ideally detected under perfect observing conditions, are instead present in the most peripheral regions of clusters, $\geq R_{200}$, which are at the edge of detectability with present (or future) instruments.

(v) The Faraday rotation measure and the radio synchrotron emission produced are significantly different in the outskirts of galaxy clusters, in cosmic filaments and in voids. Differences between models can be preferentially detected in a statistical way on large cosmic volumes (in this work we tested light cones reaching out to $z=0.8)$.

(vi) We preliminary compared with recent available tests of the cross-correlation between galaxies and diffuse synchrotron emission using MWA (Vernstrom et al. 2017) or of the RM structure function from physical pairs of radio sources (O'Sullivan et al. 2020). These tests suggest that existing surveys may already be employed to constrain primordial models of magnetism as some of the investigated models already produce too strong signals to be compatible with observations. The limited resolution available in these runs prevent us, however, from firm conclusions about which models are allowed by present radio observations.

(vii) significant differences are in principle detectable in the average deflection angle experienced by UHECRs $\left(10^{20} \mathrm{eV}\right)$ as they probe large scales of the seed magnetic field distributions in voids, which persists until $z=0$.

To summarize, in this work we have established for the first time a direct link between primordial magnetic fields (as constrained by the CMB anisotropy power spectra) and the nowadays cosmic magnetism (as it can be directly observed with radio observatories and through the propagation of charged cosmic rays). As initial conditions, we considered the most conservative Planck 2018 BICEP/KECK - SPT constraints on primordial magnetic fields, resulting from their gravitational effects on $\mathrm{CMB}$ anisotropies: the allowed comoving amplitude of the stochastic background ranges from few nano-Gauss to few pico-Gauss, depending on its scale dependence (note that tighter limits might be even obtained by considering additional effects, such as post-recombination heating, or $\mathrm{CMB}$ higher order statistics). The variation of the amplitude with the configuration is the key point in understanding the results: blue power spectra which increase the power on the smallest scales are already strongly constrained to very low amplitude by the CMB, whereas fields with more infrared spectra are allowed to larger amplitudes, yet with diluted power on much larger scales. The interplay between the scale dependence of the fields, and the constraints on their amplitude by the CMB, is perfectly reflected in our simulation results.

This research program is expected to advance on different fronts, also following the deployment of new instruments. For example future CMB experiments as LiteBIRD (Hazumi et al. 2019) ${ }^{6}$ and ground-based observatories as Simons Observatory (Ade et al. 2019) ${ }^{7}$ will further tighten the constraints on the amplitude of the fields especially on very blue spectra with Simons observatory and infrared spectra with LiteBIRD reaching the subnanoGauss and subopicoGauss level (Paoletti \& Finelli 2019).

On the theoretical side, progresses on the impact on the ionization history (Chluba et al. 2015; Kunze \& Komatsu 2015; Paoletti et al. 2019) could allow to further tighten the CMB constraints used here, which could be also made more accurate by including the modification to the matter power spectrum induced by primordial magnetic fields.

Jointly with the effort of increasing the resolution of the simulations, these advances can further bridge the connection between early- and late-cosmic magnetism to understand its origins.

\section{ACKNOWLEDGEMENTS}

The cosmological simulations described in this work were performed using the ENZO code (http://enzo-project.org). FV and SB acknowledge financial support from the ERC Starting Grant 'MAGCOW', no.714196, and the usage of computational resources on Marconi at CINECA (projects INA17_C5A38 and INA17_C4A28 with FV as Principal Investigator) and at Julich Supercomputing Centre, under project STRESSICM, as well as the usage of online storage tools kindly provided by the INAF Astronomical Archive (IA2) initiative (http://www.ia2.inaf.it). DP acknowledges the usage of computational resources on Marconi and Galileo at CINECA (project INA17_C5A42). DP and FF acknowledge financial support by ASI Grant 2016-24-H.0. MB is supported by the Deutsche Forschungsgemeinschaft (DFG, German Research Foundation) under Germany's Excellence Strategy - EXC 2121 Quantum Universe - 390833306.

\section{DATA AVAILABILITY}

The code used to produce the simulations discussed in this paper is public (enzo-project.org). The method to produce initial conditions necessary to perform the simulations are documented in the paper, and the $3 \mathrm{D}$ files can be shared by the authors upon request.

\section{REFERENCES}

Adamek J., Durrer R., Fenu E., Vonlanthen M., 2011, J. Cosmol. Astropart. Phys., 2011, 017

\footnotetext{
${ }^{6} \mathrm{~A}$ joint satellite mission JAXA-US-Europe to study the large-scale polarization of the CMB with unprecedented sensitivity with launch in 2027.

${ }^{7}$ Ground-based observatory that will observe the 40 per cent of the sky on the smallest scales with high sensitivity, will start acquiring data in 2021.
} 
Ade P. et al., 2018, preprint (arXiv:1807.02199)

Ade P. et al., 2019, J. Cosmol. Astropart. Phys., 2019, 056

Ahonen J., Enqvist K., 1998, Phys. Rev., D57, 664

Akrami Y. et al., 2018, preprint (arXiv:1810.03910)

Alves Batista R., Sigl G., 2014, J. Cosmol. Astropart. Phys., 11, 031

Alves Batista R. et al., 2016, J. Cosmol. Astropart. Phys., 5, 038

Arlen T. C., Vassilev V. V., Weisgarber T., Wakely S. P., Yusef Shafi S., 2014, ApJ, 796, 18

Ashoorioon A., Mann R. B., 2005, Phys. Rev. D, D71, 103509

Ballardini M., Finelli F., Paoletti D., 2015, J. Cosmol. Astropart. Phys., 2015, 031

Bamba K., Yokoyama J., 2004, Phys. Rev., D69, 043507

Banfi S., Vazza F., Wittor D., 2020, MNRAS, 496, 3648

Barrow J. D., Ferreira P. G., Silk J., 1997, Phys. Rev. Lett., 78, 3610

Baym G., Bodeker D., McLerran L. D., 1996, Phys. Rev. D, 53, 662

Beck A. M., Lesch H., Dolag K., Kotarba H., Geng A., Stasyszyn F. A., 2012, MNRAS, 422, 2152

Beresnyak A., Miniati F., 2016a, ApJ, 817, 127

Beresnyak A., Miniati F., 2016b, ApJ, 817, 127

Berezinsky V., 2014, Astropart. Phys., 53, 120

Bertone S., Vogt C., Enßlin T., 2006, MNRAS, 370, 319

Biermann P. L. et al., 2016, preprint (arXiv:1610.00944)

Boyanovsky D., de Vega H. J., 2005, AIP Conf. Proc. Vol. 784, MAGNETIC FIELDS IN THE UNIVERSE: From Laboratory and Stars to Primordial Structures. Am. Inst. Phys., New York, p. 434

Brandenburg A., Kahniashvili T., 2017, Phys. Rev. Lett., 118, 055102

Broderick A. E., Chang P., Pfrommer C., 2012, ApJ, 752, 22

Brüggen M., Ruszkowski M., Simionescu A., Hoeft M., Dalla Vecchia C., 2005, ApJ, 631, L21

Brunetti G., Jones T. W., 2014, Int. J. Mod. Phys. D, 23, 1430007

Brunetti G., Lazarian A., 2011, MNRAS, 410, 127

Bryan G. L. et al., 2014, ApJS, 211, 19

Burn B. J., 1966, MNRAS, 133, 67

Bykov A. M., Vazza F., Kropotina J. A., Levenfish K. P., Paerels F. B. S., 2019, Space Sci. Rev., 215, 14

Caldwell R. R., Motta L., Kamionkowski M., 2011, Phys. Rev. D, 84, 123525

Campanelli L., 2009, Int. J. Mod. Phys. D, 18, 1395

Caprini C., Gabici S., 2015, Phys. Rev. D, 91, 123514

Caprini C., Durrer R., Kahniashvili T., 2004, Phys. Rev. D, 69, 063006

Caprini C., Durrer R., Servant G., 2009, J. Cosmol. Astropart. Phys., 0912, 024

Caprioli D., Spitkovsky A., 2014, ApJ, 783, 91

Chen W., Buckley J. H., Ferrer F., 2015, Phys. Rev. Lett., 115, 211103

Chernin A. D., 1967, Sov. Astron., 10, 634

Chluba J., Paoletti D., Finelli F., Rubiño-Martín J. A., 2015, MNRAS, 451, 2244

Cho J., 2014, ApJ, 797, 133

Christensson M., Hindmarsh M., Brandenburg A., 2001, Phys. Rev. E, 64, 056405

Christensson M., Hindmarsh M., Brandenburg A., 2005, Astron. Nachr., 326, 393

Csáki C., Kaloper N., Peloso M., Terning J., 2003, J. Cosmol. Astropart. Phys., 2003, 005

Dedner A., Kemm F., Kröner D., Munz C.-D., Schnitzer T., Wesenberg M., 2002, J. Comput. Phys., 175, 645

Demozzi V., Mukhanov V., Rubinstein H., 2009, J. Cosmol. Astropart. Phys., 2009, 025

Dolag K., Bartelmann M., Lesch H., 1999, A\&A, 348, 351

Dolag K., Evrard A., Bartelmann M., 2001, A\&A, 369, 36

Dolag K., Grasso D., Springel V., Tkachev I., 2005, J. Cosmol. Astropart. Phys., 2005, 9

Dolag K., Kachelrieß M., Ostapchenko S., Tomàs R., 2009, ApJ, 703, 1078

Dolag K., Kachelriess M., Ostapchenko S., Tomàs R., 2011, ApJ, 727, L4

Donnert J., Dolag K., Lesch H., Müller E., 2009, MNRAS, 392, 1008

Donnert J., Vazza F., Brüggen M., ZuHone J., 2018, Space Sci. Rev., 214, 122

Durrer R., Caprini C., 2003, J. Cosmol. Astropart. Phys., 2003, 010

Ellis J., Fairbairn M., Lewicki M., Vaskonen V., Wickens A., 2019, J. Cosmol. Astropart. Phys., 2019, 019
Fedeli C., Moscardini L., 2012, J. Cosmol. Astropart. Phys., 11, 055

Federrath C., 2016, J. Plasma Phys., 82, 535820601

Federrath C., 2018, Phys. Today, 71, 38

Federrath C., Chabrier G., Schober J., Banerjee R., Klessen R. S., Schleicher D. R. G., 2011, Phys. Rev. Lett., 107, 114504

Federrath C., Schober J., Bovino S., Schleicher D. R. G., 2014, ApJ, 797, L19

Fenu E., Pitrou C., Maartens R., 2011, MNRAS, 414, 2354

Fidler C., Pettinari G., Pitrou C., 2016, Phys. Rev. D, 93, 103536

Field G. B., Carroll S. M., 2000, Phys. Rev. D, 62, 103008

Finelli F., Paci F., Paoletti D., 2008, Phys. Rev. D, 78, 023510

Fujita T., Namba R., Tada Y., Takeda N., Tashiro H., 2015, J. Cosmol. Astropart. Phys., 2015, 054

Gheller C., Vazza F., 2019, MNRAS, 486, 981

Gheller C., Vazza F., 2020, MNRAS, 494, 5603

Gheller C., Pantano O., Moscardini L., 1998, MNRAS, 296, 85

Giovannini M., Shaposhnikov M., 2000, Phys. Rev. D, 62, 103512

Grasso D., Riotto A., 1998, Phys. Lett. B, 418, 258

Grete P., Vlaykov D. G., Schmidt W., Schleicher D. R. G., 2016, Phys Plasmas, 23, 062317

Grete P., Vlaykov D. G., Schmidt W., Schleicher D. R. G., 2017, Phys. Rev. E, 95, 033206

Grete P., Latif M. A., Schleicher D. R. G., Schmidt W., 2019, MNRAS, 487, 4525

Guo X., Sironi L., Narayan R., 2014, ApJ, 794, 153

Haardt F., Madau P., 1996, ApJ, 461, 20

Hackstein S., Vazza F., Brüggen M., Sigl G., Dundovic A., 2016, MNRAS, 462,3660

Hackstein S., Vazza F., Brüggen M., Sorce J. G., Gottlöber S., 2018, MNRAS, , 475, 2519

Hackstein S., Brüggen M., Vazza F., Gaensler B. M., Heesen V., 2019, MNRAS, 488, 4220

Hazumi M. et al., 2019, J. Low Temp. Phys., 194, 443

Hindmarsh M., Everett A., 1998, Phys. Rev. D, 58, 103505

Hoeft M., Brüggen M., 2007, MNRAS, 375, 77

Horns D., Maccione L., Meyer M., Mirizzi A., Montanino D., Roncadelli M., 2012, Phys. Rev. D, 86, 075024

Hutschenreuter S., Dorn S., Jasche J., Vazza F., Paoletti D., Lavaux G., Enßlin T. A., 2018, Class. Quantum Gravity, 35, 154001

Iapichino L., Federrath C., Klessen R. S., 2017, MNRAS, 469, 3641

Jain R. K., Durrer R., Hollenstein L., 2014, J. Phys. Conf. Ser., 484, 012062

Jedamzik K., Saveliev A., 2019, Phys. Rev. Lett., 123, 021301

Jones T. W., Porter D. H., Ryu D., Cho J., 2011, Mem. Soc. Astron. Ital., 82, 588

Kahniashvili T., Maravin Y., Natarajan A., Battaglia N., Tevzadze A. G., 2013, ApJ, 770, 47

Kahniashvili T., Maravin Y., Lavrelashvili G., Kosowsky A., 2014, Phys. Rev. D, 90, 083004

Kahniashvili T., Brandenburg A., Tevzadze A. G., 2016, Phys. Scr., 91, 104008

Kahniashvili T., Brandenburg A., Durrer R., Tevzadze A. G., Yin W., 2017, J. Cosmol. Astropart. Phys., 1712, 002

Kanno S., Soda J., Watanabe M.-. aki ., 2009, J. Cosmol. Astropart. Phys., 0912, 009

Keisler R. et al., 2015, ApJ, 807, 151

Kronberg P. P., Lesch H., Hopp U., 1999, ApJ, 511, 56

Kulsrud R. M., Cen R., Ostriker J. P., Ryu D., 1997, ApJ, 480, 481

Kunze K. E., 2012, Phys. Rev. D, 85, 083004

Kunze K. E., Komatsu E., 2015, J. Cosmol. Astropart. Phys., 1506, 027

Langer M., Aghanim N., Puget J.-L., 2005, A\&A, 443, 367

Mack A., Kahniashvili T., Kosowsky A., 2002, Phys. Rev. D, 65, 123004

Marinacci F., Vogelsberger M., Mocz P., Pakmor R., 2015, MNRAS, 453, 3999

Matarrese S., Mollerach S., Notari A., Riotto A., 2005, Phys. Rev. D, 71, 043502

Meyer M., Conrad J., Dickinson H., 2016, ApJ, 827, 147

Miniati F., 2014, ApJ, 782, 21

Montanino D., Vazza F., Mirizzi A., Viel M., 2017, Phys. Rev. Lett., 119, 101101 
Neronov A., Vovk I., 2010, Science, 328, 73

O'Sullivan S. P. et al., 2020, MNRAS, 495, 2607

Pakmor R., Marinacci F., Springel V., 2014, ApJ, 783, L20

Paoletti D., Finelli F., 2011, Phys. Rev. D, 83, 123533

Paoletti D., Finelli F., 2013, Phys. Lett. B, 726, 45

Paoletti D., Finelli F., 2019 , J. Cosmol. Astropart. Phys., 2019, 28

Paoletti D., Chluba J., Finelli F., Rubiño-Martín J. A., 2019, MNRAS, 484, 185

Planck Collaboration XIX, 2016, A\&A, 594, A19

Pogosian L., Vachaspati T., Winitzki S., 2003, New Astron. Rev., 47, 859

Porter D. H., Jones T. W., Ryu D., 2015, ApJ, 810, 93

Quashnock J. M., Loeb A., Spergel D. N., 1989, ApJ, 344, L49

Raffelt G., Stodolsky L., 1988, Phys. Rev. D, 37, 1237

Ratra B., 1992, ApJ, 391, L1

Rieder M., Teyssier R., 2016, MNRAS, 457, 1722

Rieder M., Teyssier R., 2017, MNRAS, 471, 2674

Ryu D., Kang H., Cho J., Das S., 2008, Science, 320, 909

Ryu D., Das S., Kang H., 2010, ApJ, 710, 1422

Ryu D., Schleicher D. R. G., Treumann R. A., Tsagas C. G., Widrow L. M., 2012, Space Sci. Rev., 166, 1

Samui S., Subramanian K., Srianand R., 2017, MNRAS, 476, 1680

Sanati M., Revaz Y., Schober J., Kunze K. E., Jablonka P., 2020, A\&A, 643, 20

Saveliev A., Jedamzik K., Sigl G., 2013, Phys. Rev. D, 87, 123001

Schekochihin A. A., Cowley S. C., Taylor S. F., Maron J. L., McWilliams J. C., 2004, ApJ, 612, 276

Schekochihin A. A., Cowley S. C., Kulsrud R. M., Hammett G. W., Sharma P., 2005, ApJ, 629, 139

Schober J., Schleicher D., Federrath C., Klessen R., Banerjee R., 2012, Phys. Rev. E, 85, 026303

Semikoz V. B., Sokoloff D., 2005, A\&A, 433, L53

Seta A., Beck R., 2019, Galaxies, 7, 45

Sethi S. K., Subramanian K., 2005, MNRAS, 356, 778

Sharda P., Federrath C., Krumholz M. R., 2020, MNRAS, 497, 336

Sharma R., Subramanian K., Seshadri T. R., 2018, Phys. Rev. D, 97, 083503

Shaw J. R., Lewis A., 2010, Phys. Rev. D, 81, 043517

Shaw J. R., Lewis A., 2012, Phys. Rev. D, 86, 043510

Shu C.-W., Osher S., 1988, J. Comput. Phys., 77, 439

Sigl G., 2002, Phys. Rev. D, 66, 123002

Sigl G., Olinto A. V., Jedamzik K., 1997, Phys. Rev. D, 55, 4582

Sigl G., Miniati F., Ensslin T. A., 2003, Phys. Rev. D, 68, 043002

Sol H. et al., 2013, in Kosovichev A. G., de Gouveia Dal Pino E., Yan Y., eds, IAU Symp. 294, Solar and Astrophysical Dynamos and Magnetic Activity. Kluwer, Dordrecht, p. 459

Soler J. D., Hennebelle P., 2017, A\&A, 607, A2

Stuardi C. et al., 2020, A\&A, 638, A48

T"ornkvist O., Davis A.-C., Dimopoulos K., Prokopec T., 2001, AIP Conf. Proc. Vol. 555, Cosmology and Particle Physics: CAPP 2000. Am. Inst. Phys., New York, p. 443

Tavecchio F., Roncadelli M., Galanti G., Bonnoli G., 2012, Phys. Rev. D, 86, 085036

Tevzadze A. G., Kisslinger L., Brandenburg A., Kahniashvili T., 2012, ApJ, 759, 54

Trivedi P., Reppin J., Chluba J., Banerjee R., 2018, MNRAS, 481, 3401

Turner M. S., Widrow L. M., 1988, Phys. Rev. D, 37, 2743

Vachaspati T., 1991, Phys. Lett. B, 265, 258

Vachaspati T., 2001, Phys. Rev. Lett., 87, 251302

van Weeren R. J., de Gasperin F., Akamatsu H., Brüggen M., Feretti L., Kang H., Stroe A., Zandanel F., 2019, Space Sci. Rev., 215, 16

Vazza F., Brunetti G., Gheller C., 2009, MNRAS, 395, 1333

Vazza F., Dolag K., Ryu D., Brunetti G., Gheller C., Kang H., Pfrommer C., 2011, MNRAS, 418, 960

Vazza F., Gheller C., Brüggen M., 2014a, preprint (arXiv:1401.4454)

Vazza F., Brüggen M., Gheller C., Wang P., 2014b, MNRAS, 445, 3706

Vazza F., Ferrari C., Brüggen M., Bonafede A., Gheller C., Wang P., 2015, A\&A, 580, A119

Vazza F., Brueggen M., Gheller C., Hackstein S., Wittor D., Hinz P. M., 2017a, Class. Quantum Gravity, 34, id. 234001
Vazza F., Jones T. W., Brüggen M., Brunetti G., Gheller C., Porter D., Ryu D., 2017b, MNRAS, 464, 210

Vazza F., Brunetti G., Brüggen M., Bonafede A., 2018, MNRAS, 474, 1672

Vernstrom T., Gaensler B. M., Brown S., Lenc E., Norris R. P., 2017, MNRAS, 467,4914

Vernstrom T., Gaensler B. M., Rudnick L., Andernach H., 2019, ApJ, 878, 92

Völk H. J., Atoyan A. M., 2000, ApJ, 541, 88

Widrow L. M., 2002, Rev. Mod. Phys., 74, 775

Widrow L. M., Ryu D., Schleicher D. R. G., Subramanian K., Tsagas C. G., Treumann R. A., 2012, Space Sci. Rev., 166, 37

Wittor D., Vazza F., Brüggen M., 2017a, MNRAS, 464, 4448

Wittor D., Jones T., Vazza F., Brüggen M., 2017b, MNRAS, 471, 3212

Wittor D., Vazza F., Ryu D., Kang H., 2020, MNRAS, 495, L112

Xu H., Li H., Collins D. C., Li S., Norman M. L., 2009, ApJ, 698, L14

Zel'dovich Y. B., 1970, Sov. Astron., 13, 608

Zhang Y., Vachaspati T., Ferrer F., 2019, Phys. Rev. D, 100, 083006

Zucca A., Li Y., Pogosian L., 2017, Phys. Rev. D, 95, 063506

\section{APPENDIX A: RESOLUTION TESTS}

To test for the dependence on the spatial/mass resolution of our results, we resimulated a smaller $25^{3} \mathrm{Mpc}^{3}$ volume with the same set of cosmological parameters of the main paper, using from $128^{3}$, $256^{3}$, and $512^{3}$ cells, which gives a comoving spatial resolution of 195 (as in our main paper), 97.6 and $48.8 \mathrm{kpc}$, respectively. We limited to the simple uniform seed magnetic field model with $B_{0}=$ $2 \mathrm{nG}$, and applied the same sub-grid model for small-scale dynamo amplification as in the main paper.
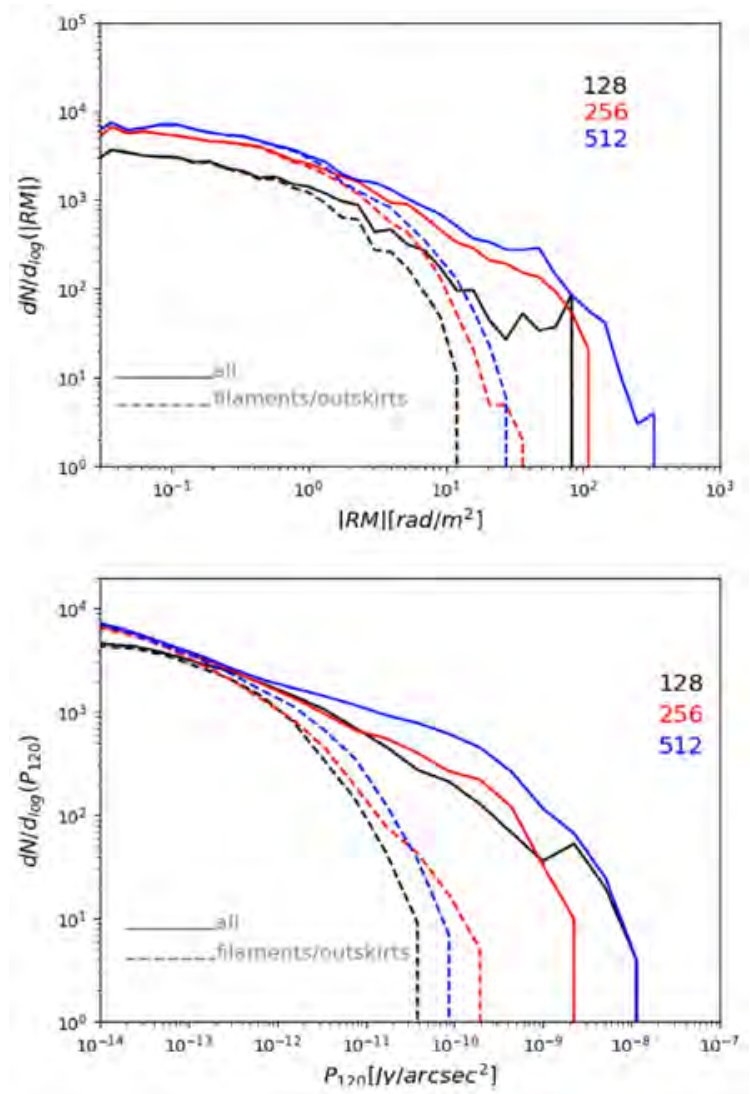

Figure A1. Distribution functions of Faraday rotation (top) and of synchrotron radio emission at $1.4 \mathrm{GHz}$ (bottom) in our resolution tests, for a $1^{\circ}$ $\times 1^{\circ}$ field of view integrated for a light cone up to $z=0.8$. 


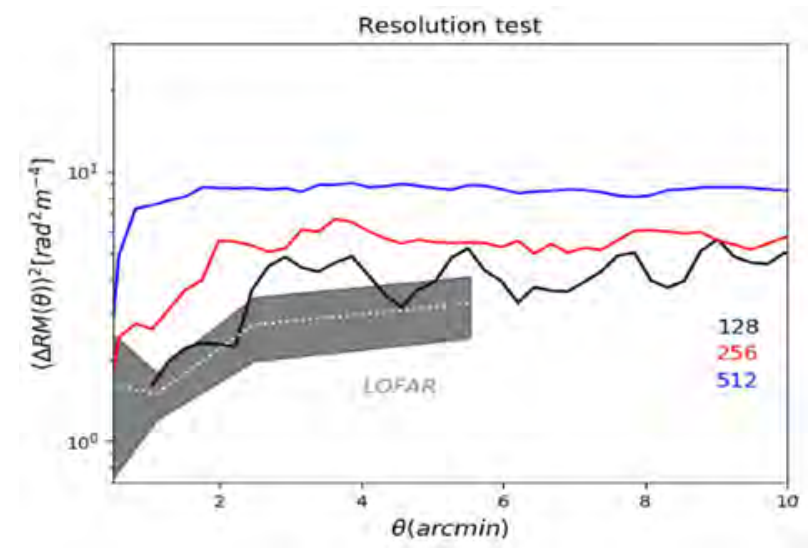

Figure A2. Simulated distribution of $\left(\Delta R M(\Delta \theta)^{2}\right)$ as a function of angular separation for our three resolution tests for a small $25^{3} \mathrm{Mpc}^{3}$ box.

Using the same approach of the main paper, we produced mock light cones of radio observables up to $z=0.8$, with the limitation that the very limited volume of this box does introduce artefacts in the final light cone, as the same structures are bound to repeat several time to fill the simulated $1^{\circ} \times 1^{\circ}$ field of view. Fig. A1 shows the distribution of integrated Faraday rotation and synchrotron emission for the light cones in the three runs. The distributions have a remarkably similar shape across resolutions, especially in the range of filaments and cluster outskirts, which is the most interesting for our study.
However, the increase of small-scale features in the gas flow internal to filaments does produce an evolution with resolution in the mock distribution of RM structure function, as shown in Fig. A2. While the 128 case looks in the range of what found in the main paper at the same resolution (albeit with a large scatter due to the small statistics of this box), the other two runs produced a higher level of $)\langle\Delta R M(\theta)\rangle)^{2}$ at most scales. Incidentally, the 256 run has a resolution close to the simulation used in our recent comparison with LOFAR data O'Sullivan et al. (2020), and once rescaled for the same initial magnetic field amplitude give a similar trend. Although not dramatic, considering the $\times 4$ increase in spatial resolution and the $\sim 4^{3}$ increased mass resolution, these resolution trends suggest that the absolute level of the RM structure function obtained in our main paper must be taken with care, due to the emergence of small-scale structures affects this observable more than others.

Fig. A3 gives the projected magnetic field strength across the simulated box, and the projected synchrotron radio emission from shocks, computed as in the main paper. The large-scale distribution of magnetic fields is remarkably similar on all scales, which also confirms that the our sub-grid dynamo model is well-behaved across resolutions, despite the progressive increase of the Reynolds number of the gas flow within galaxy clusters. On the other hand, as expected shocks are increasingly better resolved in cluster outskirts, and finer details in the morphology of synchrotron emission internal to largescale structures appear.

As a caveat, we shall notice that although these tests concerned the evolution of magnetic field topology/amplitude within filaments and cosmic structures, they cannot test the physical effects of sampling a higher $k_{D}$ (i.e. a smaller cut-off scale) in simulated primordial fields.
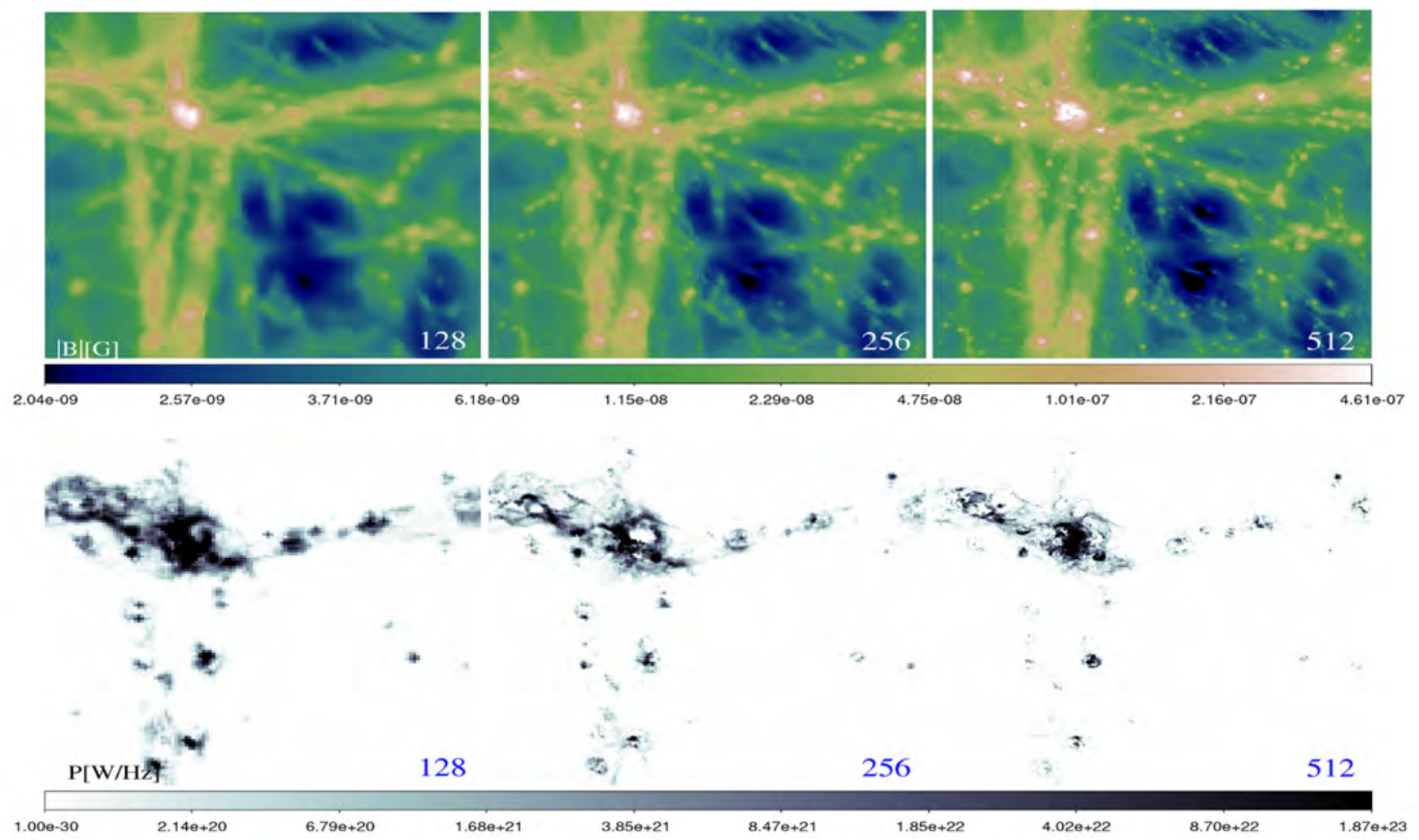

Figure A3. Maps of projected magnetic field strength (top) and synchrotron radio power (bottom) for our resolution tests at $z=0$.

This paper has been typeset from a $\mathrm{T}_{\mathrm{E}} \mathrm{X} / \mathrm{L} \mathrm{T}_{\mathrm{E}} \mathrm{X}$ file prepared by the author. 